\title{
Triterpenic Acids as Non-Competitive $\alpha$-Glucosidase Inhibitors from Boswellia elongata with Structure-Activity Relationship: In Vitro and In Silico Studies
}

\author{
Najeeb Ur Rehman ${ }^{1,+} \oplus$, Sobia Ahsan Halim ${ }^{1,+}{ }^{,}$Mohammed Al-Azri ${ }^{1}$, Majid Khan ${ }^{1,2}$, \\ Ajmal Khan ${ }^{1}$, Kashif Rafiq ${ }^{1,3}$, Ahmed Al-Rawahi ${ }^{1}$, Rene Csuk ${ }^{4}$ (D) and Ahmed Al-Harrasi ${ }^{1, *}$ \\ 1 Natural \& Medical Sciences Research Center, University of Nizwa, P.O Box 33, Birkat Al Mauz, Nizwa 616, \\ Oman; najeeb@unizwa.edu.om (N.U.R.); sobia_halim@unizwa.edu.om (S.A.H.); \\ malazri@unizwa.edu.om (M.A.-A.); majid.khan@unizwa.edu.om (M.K.); ajmalkhan@unizwa.edu.om (A.K.); \\ kashifrafiq@unizwa.edu.om (K.R.); ahmed@unizwa.edu.om (A.A.-R.) \\ 2 H. E. J. Research Institute of Chemistry, International Center for Chemical and Biological Sciences, \\ University of Karachi, Karachi 75270, Pakistan \\ 3 Department of Chemistry, Abdul Wali Khan University Mardan, Mardan 23200, Pakistan \\ 4 Organic Chemistry, Martin-Luther-University Halle-Wittenberg, Kurt-Mothes-Str. 2, D-06120 \\ Halle (Saale), Germany; rene.csuk@chemie.uni-halle.de \\ * Correspondence: aharrasi@unizwa.edu.om; Tel.: +968-25446328 \\ + Both authors equally contribute.
}

Received: 16 March 2020; Accepted: 20 April 2020; Published: 12 May 2020

Abstract: Fourteen triterpene acids, viz., three tirucallane-type (1-3), eight ursane-type (4-11), two oleanane-type $(\mathbf{1 2}, \mathbf{1 3})$ and one lupane type (21), along with boswellic aldehyde (14), $\alpha$-amyrine (15), epi-amyrine (16), straight chain acid (17), sesquiterpene (19) and two cembrane-type diterpenes $(\mathbf{1 8}, \mathbf{2 0})$ were isolated, first time, from the methanol extract of Boswellia elongata resin. Compound (1) was isolated for first time as a natural product, while the remaining compounds (2-21) were reported for first time from $B$. elongata. The structures of all compounds were confirmed by advanced spectroscopic techniques including mass spectrometry and also by comparison with the reported literature. Eight compounds (1-5, 11, 19 and 20) were further screened for in vitro $\alpha$-glucosidase inhibitory activity. Compounds $\mathbf{3}-\mathbf{5}$ and $\mathbf{1 1}$ showed significant activity against $\alpha$-glucosidase with $\mathrm{IC}_{50}$ values ranging from 9.9-56.8 $\mu \mathrm{M}$. Compound $4\left(\mathrm{IC}_{50}=9.9 \pm 0.48 \mu \mathrm{M}\right)$ demonstrated higher inhibition followed by $11\left(\mathrm{IC}_{50}=14.9 \pm 1.31 \mu \mathrm{M}\right), \mathbf{5}\left(\mathrm{IC}_{50}=20.9 \pm 0.05 \mu \mathrm{M}\right)$ and $\mathbf{3}\left(\mathrm{IC}_{50}=56.8 \pm 1.30 \mu \mathrm{M}\right)$, indicating that carboxylic acid play a key role in $\alpha$-glucosidase inhibition. Kinetics studies on the active compounds 3-5 and $\mathbf{1 1}$ were carried out to investigate their mechanism (mode of inhibition and dissociation constants $K_{i}$ ). All compounds were found to be non-competitive inhibitors with $K_{i}$ values in the range of 7.05 $\pm 0.17-51.15 \pm 0.25 \mu \mathrm{M}$. Moreover, in silico docking was performed to search the allosteric hotspot for ligand binding which is targeted by our active compounds investigates the binding mode of active compounds and it was identified that compounds preferentially bind in the allosteric binding sites of $\alpha$-glucosidase. The results obtained from docking study suggested that the carboxylic group is responsible for their biologic activities. Furthermore, the $\alpha$-glucosidase inhibitory potential of the active compounds is reported here for the first time.

Keywords: Boswellia elongata; triterpene acids; $\alpha$-glucosidase inhibitors; NMR spectroscopy; kinetics study; homology modeling; molecular docking 


\section{Introduction}

Diabetes mellitus (DM) - mostly characterized by high blood-glucose levels (hyperglycemia), and their complications-increases the morbidity and mortality threats for type-2 diabetes patients [1,2]. Poor control of the post-prandial glucose levels, mostly concerned with type-2 DM, leads to atherosclerosis and cardiovascular disorders [3,4]. It has been reported that about $90 \%$ of the world's diabetic people have Type-2 DM [5]. $\alpha$-Glucosidase inhibitors (AGIs) have inadequate protection, temporally recover the blood glucose levels, and improve Type-2 DM complications, together with the treatment of obesity [6,7] but accomplished with gastrointestinal side-effects like diarrhea, flatulence and abdominal discomfort [8-10].

Natural products are known to have anti-diabetic effects and offered plentiful exciting potentials for the future development and improvement of successful therapies [6]. Interesting previously isolated boswellic acids (BAs), bioactive components of frankincense, from the resins of Boswellia sacra and B. papyrifera demonstrated promising $\alpha$-glucosidase activity [11]. Keeping in mind the side effects of the existing synthetic drugs and especially a crucial role of $\alpha$-glucosidase enzyme in hyperglycemia, there is an urgent need to discover safe and effective enzyme inhibitors as an approach to effectively control the diabetic disorders.

The genus Boswellia (Burseraceae) consists of 23 species widespread throughout the world, mainly in Arabia, in eastern coast of Africa and in India [12,13]. Frankincense (olibanum), gum resin obtained from trees of the genus Boswellia, is mostly used in traditional remedies for decades [14] against fevers, dysentery, antiseptic and as an antitumor agent [15,16]. BAs (bioactive components of frankincense) are mostly isolated from the resins of Boswellia species and considered to have interesting pharmacological, biologic and medicinal applications against chronic colitis, asthma, inflammation, arthritis, stomach ache, ulcerative colitis and hepatitis [17-19].

Eight species of the genus are available in Soqotra Island. Boswellia elongate Balf. $\mathrm{f}$. (endemic to Soqotra) is mostly found on stony soils with valuable producing frankincense [20]. B. elongata, one of the most important Soqotraen medicinal plants, is used traditionally to treat common cold, bronchitis, relieving fever and pain, sweetening the breath, sooth a disturb stomach, rheumatism as well as a remedy for asthma [21]. Different parts of the plant are useful in a variety of diseases like diarrhea, urinary disorder, dysentery, gonorrhea, bronchitis [13]. The essential oil of B. elongata was dominated by verticillol, $\beta$-caryophyllene and methyl cycloundecanecarboxylate having 52.4\%, 39.1\% and 7.9\%, respectively [12]. Previous biologic investigation on the bark of $B$. elongata reported significant antimicrobial and antiviral activities [21,22]. Previous report on the analgesic and anti-inflammatory activities of methanolic extract further supported the traditional application of this plant in treating various diseases associated with inflammation and pain [13]. However, no report is available on the phytochemical investigations of the title resin.

\section{Experimental}

\subsection{General}

High-resolution electrospray ionisation mass spectrometry (HR-ESI-MS) spectra were recorded on Agilent 6530 LC Q-TOF (country of origin USA/EU, made in Singapore). Infra-red (IR) spectra were recorded on a ATR-Tensor 37 spectrometer, Bruker (Ettlingen, Baden-Württemberg, Germany) with wave numbers $(v)$ in $\mathrm{cm}^{-1}$. Optical rotations were measured on a KRUSS P3000 polarimeter (A. Kruss Optronic, Hamburg, Germany). The ${ }^{1} \mathrm{H}$ - and ${ }^{13} \mathrm{C}$ NMR spectra were recorded on nuclear magnetic resonance (NMR) spectrometer (Bruker, Zürich, Switzerland) operating at $600 \mathrm{MHz}(150 \mathrm{MHz}$ for $\left.{ }^{13} \mathrm{C}\right)$ using the solvent peaks as internal references $\left(\mathrm{CDCl}_{3}, \delta_{\mathrm{H}}: 7.26 ; \delta_{\mathrm{C}}: 77.0\right),\left(\mathrm{CD}_{3} \mathrm{OD}, \delta_{\mathrm{H}}: 4.87 ; \delta_{\mathrm{C}}\right.$ : 48.5). Data were reported in the following order: chemical shift $(\delta)$ in ppm; multiplicities are indicated $\mathrm{s}=$ singlet, $\mathrm{d}=$ doublet, $\mathrm{t}=$ triplet, $\mathrm{dd}=$ doublet of doublet, $\mathrm{m}=$ multiplet; coupling constants $(\mathrm{J})$ are in hertz $(\mathrm{Hz})$. Column chromatography was carried out by using silica gel of the selected particle size of 100-200 mesh. For thin layer chromatography TLC, pre-coated aluminum sheets (silica gel 60F-254, 
Merck, Darmstadt, Hesse, Germany) were used. Visualization was accomplished with UV-light (254 and $366 \mathrm{~nm}$ ) or $\mathrm{I}_{2}$ stain and also by spraying with the ceric sulfate reagent.

\subsection{Plant Material and Identification}

The gum resin of B. elongata was donated by Mr. Mohammed Khalifa (Yemen, 2017) and identified by Dr. Labib Noman from Island of Socotra. The voucher specimen (BEL/04/2017) of the sample was deposited in the herbarium of the Natural \& Medical Sciences Research Center, University of Nizwa, Oman.

\subsection{Extraction and Isolation}

The air-dried powder resin of B. elongata (100 g) was finely extracted with $\mathrm{MeOH}(1 \mathrm{~L})$ at room temperature (three times) and evaporated under reduced pressure to yield a yellow semi-solid methanol residue $(66.0 \mathrm{~g})$. The crude $\mathrm{MeOH}$ extract was successively fractionated into $n$-hexane $(12.7 \mathrm{~g})$, ethyl acetate $(47.5 \mathrm{~g})$ and aqueous $(6.0 \mathrm{~g})$. The $n$-hexane fraction was first subjected to column chromatography using 5\%,10\%, 20\%, 30\% and 50\% EtOAc/n-hexane to afford twenty-two fractions $\left(\mathrm{BEHF}_{1-22}\right)$. Fractions $\left(\mathrm{BEHF}_{5-10}, 10 \%-20 \%\right.$-hexane/EtOAc) were further subjected to silica gel column chromatography (CC) one by one using an isocratic mobile phase to get compounds 14-21.

Similarly, ethyl acetate fraction was subjected to CC using isocratic mobile phase viz., 10\%, 20\%, $30 \%, 40 \%$ and $50 \% \mathrm{AcOEt} / n$-hexane to afford sixteen fractions $\left(\mathrm{BEEF}_{1-16}\right)$. After taking TLC, sub fraction $\left(\mathrm{BEEF}_{4}\right)$ was further chromatographed on $\mathrm{CC}$ to afford three compounds 1 (5.7 mg), 2 (14.6 mg) and 3 (3.5 mg) using 20\% and 30\% AcOEt/n-hexane system as a mobile phase along with some semi-pure compounds 10 (10.5 mg) and $11(17 \mathrm{mg})$, which were later on purified through preparative high performance liquid chromatography (HPLC) using $\mathrm{CHCl}_{3}$ solvent. Sub fractions $\mathrm{BEEF}_{6-10}$ were combined due to their similar TLC profile and further subjected on CC using $n$-hexane/AcOEt with increasing polarity $(2: 8,4: 6,6: 4$ and 8:2) to afford compounds 4-9.

\section{4. $\alpha$-Tirucalla-8, 24-Dien-3 $\alpha$-Acetate (1)}

Compound 1: colorless solid; UV $(\mathrm{MeOH}) \lambda_{\max } 218(3.38) ;[\alpha]^{25} \mathrm{D} 17.8^{\circ}(\mathrm{MeOH}, \mathrm{c}=0.17) ; \mathrm{IR}$ (solid) $v_{\max }$ $1724\left(\mathrm{CH}_{3} \mathrm{CO}\right), 1624$ (C=C), 1446, 1366, 1215, 1020, $920 \mathrm{~cm}^{-1} ;{ }^{1} \mathrm{HNMR}\left(\mathrm{CDCl}_{3}, 600 \mathrm{MHz}\right): \delta 5.08(1 \mathrm{H}$, $\mathrm{t}=7.2,5.4 \mathrm{~Hz}, \mathrm{H}-24), 4.49(1 \mathrm{H}, \mathrm{dd}, \mathrm{J}=12.0,4.8 \mathrm{~Hz}, \mathrm{H}-3), 2.02(3 \mathrm{H}, \mathrm{s}, \mathrm{H}-32), 1.65(3 \mathrm{H}, \mathrm{s}, \mathrm{H}-26), 1.58(3 \mathrm{H}$, s, H-27), 1.62 (1H, br. s, H-5), 1.46 (1H, m. H-17), 1.39 (1H, m, H-20), 0.95 (3H, s, H-28), 0.93 (3H, d, $J=6.4 \mathrm{~Hz}, \mathrm{H}-21), 0.89$ (3H, s, H-19), 0.85 (6H, s, H-29/30), 0.72 (3H, s, H-18); ${ }^{13} \mathrm{C}-\mathrm{NMR}\left(\mathrm{CDCl}_{3}, 125\right.$ MHz): $\delta 171.0$ (C-31), 133.9 (C-9), 133.6 (C-8), 131.2 (C-25), 125.2 (C-24), 80.9 (C-31), 51.0 (C-5), 50.1 (C-17), 49.9 (C-14), 44.0 (C-13), 37.8 (C-4), 37.1 (C-10), 36.3 (C-22), 36.2 (C-20), 34.9 (C-7), 30.8 (C-12), 29.8 (C-15), 28.0 (C-28), 27.9 (C-16), 27.5 (C-2), 25.7 (C-27), 24.9 (C-23), 24.3 (C-1), 24.2 (C-30), 21.4 (C-11), 21.3 (C-32), 20.1 (C-19), 18.7 (C-6), 18.6 (C-29/21), 16.6 (C-26), 15.3 (C-18); HRMS (ESI+): m/z 469.3292 $[\mathrm{M}+\mathrm{H}]^{+}$(calculated for $\left.\mathrm{C}_{32} \mathrm{H}_{53} \mathrm{O}_{2}: 469.3280\right)$.

$5 \alpha$-Tirucalla-8,24-dien-3 $\alpha$-ol (2): colorless solid; ${ }^{1} \mathrm{H}-\mathrm{NMR}(600 \mathrm{MHz}$, chloroform- $d): \delta 5.08(1 \mathrm{H}, \mathrm{brt}, 5.4$, $1.2 \mathrm{~Hz}), 3.41(1 \mathrm{H}$, br.t, $3.0 \mathrm{~Hz}), 1.66(3 \mathrm{H}, \mathrm{s}), 1.56(3 \mathrm{H}, \mathrm{s}), 0.94(3 \mathrm{H}, \mathrm{s}), 0.93(3 \mathrm{H}, \mathrm{s}), 0.89(3 \mathrm{H}, \mathrm{d}, \mathrm{J}=6.0 \mathrm{~Hz})$, 0.84 (6H, br.s), $0.74(3 \mathrm{H}, \mathrm{s}) ;{ }^{13} \mathrm{C}-\mathrm{NMR}(150 \mathrm{MHz}$, chloroform- $d): \delta 15.5,17.6,18.6,18.8,19.9,21.4,22.2$, 24.4, 24.9, 25.7, 25.8, 27.2, 28.0, 28.0, 29.7, 30.8, 36.3, 36.4, 37.1, 37.6, 44.0, 44.8, 55.0, 55.0, 125.2, 130.8, 133.3, 134.2; HRMS (ESI+): $m / z 426.9714[\mathrm{M}+\mathrm{H}]^{+}$.

$3 \alpha$-Acetoxytirucall-8,24-dien-21-oic acid (3): colorless solid; ${ }^{1} \mathrm{H}-\mathrm{NMR}(600 \mathrm{MHz}$, chloroform-d): $\delta 5.08$ (1H, br.t, 7.2, $6.6 \mathrm{~Hz}), 4.63$ (1H, br.s), 2.02 (3H, s, Ac), $1.65(3 \mathrm{H}, \mathrm{s}), 1.56(3 \mathrm{H}, \mathrm{s}), 1.24(3 \mathrm{H}, \mathrm{s}), 0.91$ (3H, s), $0.88(3 \mathrm{H}, \mathrm{s}), 0.86(3 \mathrm{H}, \mathrm{s}), 0.84(3 \mathrm{H}, \mathrm{s}) ;{ }^{13} \mathrm{C}-\mathrm{NMR}(150 \mathrm{MHz}$, chloroform-d $): \delta 15.8,17.6,18.5,19.8$, 21.3, 21.4, 21.8, 23.3, 24.4, 25.6, 25.9, 26.9, 27.0, 27.6, 27.0, 27.6, 28.8, 29.3, 29.6, 30.5, 32.4, 36.7, 37.1,

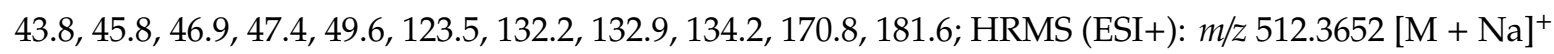
$\left(\mathrm{C}_{35} \mathrm{H}_{56} \mathrm{O}_{5} \mathrm{Na}\right)$. 
3-O-Acetyl-9,11-dehydro- $\beta$-boswellic acid (4): colorless solid; ${ }^{1} \mathrm{H}-\mathrm{NMR}(600 \mathrm{MHz}$, chloroform- $d$ ); $\delta$ $5.63(1 \mathrm{H}, \mathrm{d}, J=6.0 \mathrm{~Hz}), 5.44(1 \mathrm{H}, \mathrm{d}, J=5.4 \mathrm{~Hz}), 5.27(1 \mathrm{H}, \mathrm{br} . \mathrm{s}), 2.02(3 \mathrm{H}, \mathrm{s}, \mathrm{Ac}), 1.24(3 \mathrm{H}, \mathrm{s}), 1.19(3 \mathrm{H}$, s), $1.09(3 \mathrm{H}, \mathrm{s}), 0.92(3 \mathrm{H}, \mathrm{s}), 0.90(3 \mathrm{H}, \mathrm{s}), 0.84(3 \mathrm{H}, \mathrm{s}), 0.78(3 \mathrm{H}, \mathrm{d}, J=5.4 \mathrm{~Hz}) ;{ }^{13} \mathrm{C}-\mathrm{NMR}(150 \mathrm{MHz}$, chloroform-d): 17.3, 17.4, 19.5, 21.2, 21.5, 21.7, 23.2, 23.7, 24.3, 26.1, 28.2, 28.7, 31.1, 31.8, 33.1, 33.6, 39.0, 39.0, 39.4, 40.6, 41.3, 43.3, 46.9, 47.4, 57.3, 72.9, 116.5, 123.0, 141.6, 152.4, 170.3, 182.2; HRMS (ESI+): $\mathrm{m} / \mathrm{z}$ $497.3655[\mathrm{M}+\mathrm{H}]^{+}\left(\mathrm{C}_{35} \mathrm{H}_{56} \mathrm{O}_{5} \mathrm{Na}\right)$

9,11-Dehydro- $\beta$-boswellic acid (5): colorless solid material; ${ }^{1} \mathrm{H}-\mathrm{NMR}(600 \mathrm{MHz}$, chloroform- $d$ ); $\delta 5.63$ $(1 \mathrm{H}, \mathrm{d}, J=5.4 \mathrm{~Hz}), 5.43(1 \mathrm{H}, \mathrm{d}, J=5.4 \mathrm{~Hz}), 4.06(1 \mathrm{H}, \mathrm{br} . \mathrm{t}), 1.34(3 \mathrm{H}, \mathrm{s}), 1.16(3 \mathrm{H}, \mathrm{s}), 1.08(3 \mathrm{H}, \mathrm{s}), 0.90(6 \mathrm{H}$, s), 0.83 (3H, br.s), 0.78 (3H, d, $J=6.6 \mathrm{~Hz}) ;{ }^{13} \mathrm{C}-\mathrm{NMR}(150 \mathrm{MHz}$, chloroform-d): $\delta 17.3,17.5,19.6,21.5$, 21.7, 23.3, 24.1, 26.1, 26.9, 28.2, 28.7, 31.2, 31.8, 32.5, 33.6, 39.0, 39.1, 39.4, 40.6, 41.3, 43.3, 46.0, 47.6, 57.3, 70.3, 116.4, 122.9, 141.5, 152.7, 183.0; HRMS (ESI+): $m / z$ 455.3516 [M + H] $]^{+}$.

3-Acetyl- $\beta$-boswellic acid ( $\beta$-ABA, 6): colorless needles; ${ }^{1} \mathrm{H}$ NMR $(600 \mathrm{MHz}$, chloroform- $d) 5.28(1 \mathrm{H}$, brt, H-12), 5.12 (1H, br.t, H-3), 2.07 (3H, s, H-3, Ac), $1.24(3 \mathrm{H}, \mathrm{s}), 1.16(3 \mathrm{H}, \mathrm{s}), 1.02(3 \mathrm{H}, \mathrm{s}), 0.90(3 \mathrm{H}, \mathrm{s})$ $0.87(3 \mathrm{H}, \mathrm{s}), 0.81(3 \mathrm{H}, \mathrm{d}, 6.6 \mathrm{~Hz}), 0.78(3 \mathrm{H}, \mathrm{d}, J=6.6 \mathrm{~Hz}) ;{ }^{13} \mathrm{C}$ NMR $(150 \mathrm{MHz}$, chloroform- $d): \delta 13.3,16.8$, 17.4, 19.6, 21.3, 21.3, 23.2, 23.4, 23.6, 23.6, 26.5, 28.1, 28.7, 29.6, 31.2, 33.0, 33.8, 34.5, 37.4, 39.6, 39.7, 40.0, $41.5,42.2,46.5,46.8,50.5,59.1,73.2,124.5,139.5,170.3,181.2$; HRMS (ESI+): $m / z 497.3918$ [M + H] ${ }^{+}$.

3-Acetyl 11-keto- $\beta$-boswellic acid (AKBA, 7): ${ }^{1} \mathrm{H}-\mathrm{NMR}(600 \mathrm{MHz}$, chloroform- $d$ ): $\delta 5.53(1 \mathrm{H}$, br.s, $\mathrm{H}-12), 5.27(1 \mathrm{H}, \mathrm{t}, \mathrm{H}-3), 2.38(1 \mathrm{H}, \mathrm{s}, \mathrm{H}-9), 2.06(3 \mathrm{H}, \mathrm{s}, \mathrm{H}-3 \mathrm{Ac}), 1.30(3 \mathrm{H}, \mathrm{s}), 1.21(3 \mathrm{H}, \mathrm{s}), 1.16(3 \mathrm{H}, \mathrm{s}), 1.11$ $(3 \mathrm{H}, \mathrm{s}), 0.92(3 \mathrm{H}, \mathrm{s}), 0.80(3 \mathrm{H}, \mathrm{d}, J=7.2), 0.79(3 \mathrm{H}, \mathrm{d}, J=6.0) ;{ }^{13} \mathrm{C}-\mathrm{NMR}(150 \mathrm{MHz}$, chloroform- $d): \delta$ 13.2, 14.1, 17.4, 18.3, 18.7, 20.5, 21.0, 21.1, 21.3, 23.5, 23.8, 27.2, 27.5, 28.8, 30.9, 32.8, 33.9, 34.6, 37.3, 39.2, 39.3, 40.9, 43.7, 45.0, 46.4, 50.3, 59.0, 60.3, 60.4, 73.1, 130.4, 164.9, 170.2, 180.8, 199.3; HRMS (ESI+): $\mathrm{m} / \mathrm{z}$ $513.3568[\mathrm{M}+\mathrm{H}]+$.

$\beta$-Boswellic acid ( $\beta$-BA, 8): colorless solid; ${ }^{1} \mathrm{H}-\mathrm{NMR}(600 \mathrm{MHz}$, chloroform- $d) ; \delta 5.11(1 \mathrm{H}$, br.t), 4.08 (1H, br.s), $1.31(3 \mathrm{H}, \mathrm{s}), 1.12(3 \mathrm{H}, \mathrm{s}), 1.03(3 \mathrm{H}, \mathrm{s}), 0.88(6 \mathrm{H}, \mathrm{s}), 0.81(3 \mathrm{H}, \mathrm{d}, \mathrm{J}=6.6 \mathrm{~Hz}), 0.76(3 \mathrm{H}, \mathrm{d}, \mathrm{J}=6.0$ $\mathrm{Hz}) ;{ }^{13} \mathrm{C}-\mathrm{NMR}(150 \mathrm{MHz}$, chloroform- $d$ ): $\delta 13.3,16.8,17.4,19.7,21.3,23.2,23.4,24.1,26.2,26.5,28.1$, 28.7, 31.2, 31.1, 33.8, 33.9, 37.5, 39.6, 39.7, 40.0, 41.5, 42.3, 46.8, 47.3, 49.1, 59.5, 70.7, 124.5, 139.6, 182.6; HRMS (ESI'): $m / z 455.3996[\mathrm{M}-\mathrm{H}]^{+}$.

11-Keto- $\beta$-boswellic acid (KBA, 9): colorless crystals; ${ }^{1} \mathrm{H}-\mathrm{NMR}(600 \mathrm{MHz}$, chloroform- $d$ ); $\delta 5.52(1 \mathrm{H}, \mathrm{s})$, 4.05 (br.t), $1.31(3 \mathrm{H}, \mathrm{s}), 1.28(3 \mathrm{H}, \mathrm{s}), 1.15(3 \mathrm{H}, \mathrm{s}), 1.09(3 \mathrm{H}, \mathrm{s}), 0.90(3 \mathrm{H}, \mathrm{s}), 0.79(3 \mathrm{H}, \mathrm{d}, J=6.0 \mathrm{~Hz}), 0.77$ $(3 \mathrm{H}, \mathrm{d}, J=6.6 \mathrm{~Hz}) ;{ }^{13} \mathrm{C}-\mathrm{NMR}(150 \mathrm{MHz}$, chloroform- $d$ ): $\delta 13.2,17.4,18.4,18.8,20.5,21.1,24.3,26.2,27.2$, 27.5, 28.9, 30.9, 32.9, 33.9, 34.0, 37.5, 39.3, 39.3, 40.9, 43.8, 45.1, 47.2, 48.8, 59.0, 60.4, 70.5, 130.5, 165.0, 182.0, 199.6; HRMS (ESI+): $m / z 471.3473[\mathrm{M}+\mathrm{H}]^{+}$.

$3 \alpha, 11 \alpha$-dihydroxyurs-12-en-24-oic acid (10): white solid; ${ }^{1} \mathrm{H}-\mathrm{NMR}(600 \mathrm{MHz}$, chloroform- $d$ ); $\delta 5.16$ $(1 \mathrm{H}$, br.t), $4.22(1 \mathrm{H}, \mathrm{d}$, br.t, J = 9.6, $6.0 \mathrm{~Hz}), 3.98(1 \mathrm{H}$, br.t), $1.27(3 \mathrm{H}, \mathrm{s}), 1.24(3 \mathrm{H}, \mathrm{s}), 1.14(3 \mathrm{H}, \mathrm{s}), 1.08(3 \mathrm{H}$, s), $0.96(6 \mathrm{H}, \mathrm{s}), 0.84(3 \mathrm{H}, \mathrm{d}, \mathrm{J}=3.0 \mathrm{~Hz}) ;{ }^{13} \mathrm{C}-\mathrm{NMR}(150 \mathrm{MHz}$, chloroform- $d): \delta 14.8,18.1,18.7,20.9,21.7$, 23.3, 25.3, 27.3, 27.6, 29.1, 29.3, 32.2, 34.8, 35.3, 37.2, 39.8, 40.8, 40.9, 42.6, 43.4, 44.5, 54.4, 59.8, 69.4, 71.6, 131.2, 142.6, 181.4; HRMS (ESI+): $m / z 455.3463[\mathrm{M}+\mathrm{H}]^{+}$.

$11 \alpha$-methoxy- $\beta$-boswellic acid (11): white solid; ${ }^{1} \mathrm{H}-\mathrm{NMR}(600 \mathrm{MHz}$, chloroform- $d$ ); $\delta 5.29(1 \mathrm{H}, \mathrm{s}), 4.03$ $(1 \mathrm{H}, \mathrm{s}), 3.87(1 \mathrm{H}, \mathrm{dd}, J=9.0,2.4 \mathrm{~Hz}), 1.38(3 \mathrm{H}, \mathrm{s}), 1.28(3 \mathrm{H}, \mathrm{s}), 1.14(3 \mathrm{H}, \mathrm{s}), 1.07(3 \mathrm{H}, \mathrm{s}), 0.98(3 \mathrm{H}, \mathrm{d}$, $J=6.6 \mathrm{~Hz}), 0.87(3 \mathrm{H}, \mathrm{d}, J=6.4 \mathrm{~Hz}), 0.77(3 \mathrm{H}, \mathrm{s}) ;{ }^{13} \mathrm{C}-\mathrm{NMR}(150 \mathrm{MHz}$, chloroform- $d): \delta 14.3,17.4,18.2$, 19.5, 21.3, 22.5, 24.5, 26.5, 26.6, 27.9, 28.7, 31.1, 33.7, 33.8, 34.9, 38.7, 39.3, 39.5, 41.3, 42.3, 42.9, 47.6, 49.0, 50.6, 54.1, 58.7, 77.0, 70.8, 124.4, 143.5, 183.0; HRMS (ESI+): $\mathrm{m} / \mathrm{z} 455.3563[\mathrm{M}+\mathrm{H}]^{+}$.

3-Acetyl- $\alpha$-boswellic acid ( $\alpha$-ABA, 12): colorless needles; ${ }^{1} \mathrm{H}$-NMR $(600 \mathrm{MHz}$, chloroform- $d$ ); $\delta 5.28$ (1H, brt), $5.17(1 \mathrm{H}, \mathrm{brt}), 2.05(3 \mathrm{H}, \mathrm{s}, \mathrm{Ac}), 1.16(3 \mathrm{H}, \mathrm{s}), 1.12(3 \mathrm{H}, \mathrm{s}), 0.97(3 \mathrm{H}, \mathrm{s}), 0.88(3 \mathrm{H}, \mathrm{s}), 0.84(6 \mathrm{H}, \mathrm{s})$, $0.77(3 \mathrm{H}, \mathrm{s}) ;{ }^{13} \mathrm{C}-\mathrm{NMR}(150 \mathrm{MHz}$, chloroform- $d$ ): $\delta 13.2,16.8,19.7,21.3,23.5,23.6,23.7,25.9,26.1,27.0$, 28.4, 29.7, 31.1, 32.5, 32.8, 33.3, 34.4, 34.7, 37.1, 37.4, 39.8, 41.9, 46.4, 46.8, 46.9, 50.5, 73.7, 121.9, 145.1, 170.3, 178.2; HRMS (ESI'): $m / z 497.3918[\mathrm{M}-\mathrm{H}]^{+}$. 
$\alpha$-Boswellic acid ( $\alpha$-BA, 13): white solid; ${ }^{1} \mathrm{H}-\mathrm{NMR}(600 \mathrm{MHz}$, chloroform- $d$ ); $\delta 5.16(1 \mathrm{H}, \mathrm{br} . \mathrm{t}), 4.05$ (1H, br.t), $1.31(3 \mathrm{H}, \mathrm{s}), 1.27(3 \mathrm{H}, \mathrm{s}), 1.12(3 \mathrm{H}, \mathrm{s}), 1.05(3 \mathrm{H}, \mathrm{s}), 0.94(3 \mathrm{H}, \mathrm{s}), 0.87(6 \mathrm{H}, \mathrm{d}, \mathrm{J}=3.0 \mathrm{~Hz}), 0.76(3 \mathrm{H}, \mathrm{s})$; ${ }^{13} \mathrm{C}-\mathrm{NMR}(150 \mathrm{MHz}$, chloroform- $d$ ): $\delta$ 13.2, 16.7, 19.8, 23.6, 23.7, 24.1, 26.0, 26.0, 26.2, 27.0, 28.4, 29.7, $31.1,32.5,32.8,33.3,33.8,34.7,37.2,37.6,41.9,46.8,46.8,47.2,47.3,49.0,71.0,121.8,145.1$, 179.5; HRMS $\left(\mathrm{ESI}^{-}\right): \mathrm{m} / \mathrm{z} 455.3748[\mathrm{M}-\mathrm{H}]^{+}$.

$\beta$-Boswellic aldehyde (14): colorless solid; ${ }^{1} \mathrm{H}-\mathrm{NMR}(600 \mathrm{MHz}$, chloroform- $d) ; \delta 9.75(1 \mathrm{H}, \mathrm{s}), 5.14(1 \mathrm{H}$, br.t), 4.15 (1H, br.t), $1.27(3 \mathrm{H}, \mathrm{s}), 1.24(3 \mathrm{H}, \mathrm{s}), 1.10(3 \mathrm{H}, \mathrm{s}), 1.02(3 \mathrm{H}, \mathrm{s}), 0.97(3 \mathrm{H}, \mathrm{s}), 0.88(6 \mathrm{H}, \mathrm{d}, \mathrm{J}=6.6$ $\mathrm{Hz}), 0.77(3 \mathrm{H}, \mathrm{s}) ;{ }^{13} \mathrm{C}-\mathrm{NMR}(150 \mathrm{MHz}$, chloroform- $d)$ : $\delta 14.2,17.0,17.4,17.8,19.6,21.3,23.2,23.5,25.9$, 26.5, 28.0, 28.7, 29.6, 31.2, 33.1, 33.2, 33.7, 37.2, 39.5, 39.6, 40.0, 41.5, 42.3, 46.3, 49.2, 52.2, 59.1, 69.3, 124.4, 139.6, 205.1; HRMS (ESI+): $m / z 441.3726[\mathrm{M}+\mathrm{H}]^{+}$.

epi- $\alpha$-Amyrin (15): white amorphous powder; ${ }^{1} \mathrm{H}-\mathrm{NMR}(600 \mathrm{MHz}$, chloroform- $d): \delta 5.11(1 \mathrm{H}, \mathrm{t}, J=4.8$ $\mathrm{Hz}), 3.38$ (1H, Brs), $1.06(3 \mathrm{H}, \mathrm{s}), 0.98(3 \mathrm{H}, \mathrm{s}), 0.94(6 \mathrm{H}, \mathrm{s}), 0.89(3 \mathrm{H}, \mathrm{d}, 5.4 \mathrm{~Hz}), 0.84(3 \mathrm{H}, \mathrm{s}), 0.77(3 \mathrm{H}, \mathrm{s})$, $0.76(3 \mathrm{H}, \mathrm{d}=\mathrm{J}=5.6 \mathrm{~Hz}) ;{ }^{13} \mathrm{C}-\mathrm{NMR}(150 \mathrm{MHz}$, chloroform-d): $\delta 15.4,16.8,17.4,18.2,21.4,22.3,23.2$, 23.3, 25.2, 26.5, 28.1, 28.2, 28.7, 31.2, 32.8, 33.2, 33.7, 36.9, 37.3, 39.6, 39.6, 40.1, 41.5, 42.1, 76.1, 124.4, 139.5; HRMS (ESI+): $m / z 426.9669[\mathrm{M}+\mathrm{H}]^{+}$.

$\alpha$-Amyrin (16): colorless powder; ${ }^{1} \mathrm{H}-\mathrm{NMR}(600 \mathrm{MHz}$, chloroform- $d) ; \delta 5.11(1 \mathrm{H}, \mathrm{t}, J=3.6 \mathrm{~Hz}), 3.21-3.18$ $(\mathrm{dd}, J=4.8 \mathrm{~Hz}), 1.05(3 \mathrm{H}, \mathrm{s}), 0.98(3 \mathrm{H}, \mathrm{s}), 0.97(3 \mathrm{H}, \mathrm{s}), 0.93(3 \mathrm{H}, \mathrm{s}), 0.88(3 \mathrm{H}, \mathrm{s}), 0.77(3 \mathrm{H}, \mathrm{s}), 0.76(6 \mathrm{H}, \mathrm{d}$, $4.8 \mathrm{~Hz}) ;{ }^{13} \mathrm{C}-\mathrm{NMR}(150 \mathrm{MHz}$, chloroform- $d): \delta 15.6,15.6,16.8,17.4,18.0,18.3,21.4,23.2,23.3,26.6,27.2$, 27.9, 28.1, 28.7, 31.2, 32.9, 33.7, 36.8, 38.7, 39.6, 39.6, 40.0, 41.5, 42.0, 47.7, 55.1, 59.0, 79.0, 124.4, 139.5; HRMS (ESI+): $m / z 426.9673[\mathrm{M}+\mathrm{H}]^{+}$.

Tricosanoic acid (17): colorless solid; ${ }^{1} \mathrm{H}-\mathrm{NMR}(600 \mathrm{MHz}$, chloroform- $d)$; $\delta 2.33(2 \mathrm{H}, \mathrm{t}, 7.8 \mathrm{~Hz}), 1.62(4 \mathrm{H}$, m), 1.60-1.08 $\left(\mathrm{CH}_{2}\right)_{18}, 0.94$ (3H. m); ${ }^{13} \mathrm{C}-\mathrm{NMR}(150 \mathrm{MHz}$, chloroform-d): $\delta 14.1,22.6,24.7,29.0,29.23$, 29.3, 29.4, 29.5, 31.9, 33.6, 177.8; HRMS (ESI+): $m / z 355.2451[\mathrm{M}+\mathrm{H}]^{+}$.

Incensole (18): colorless oil; ${ }^{1} \mathrm{H}-\mathrm{NMR}(600 \mathrm{MHz}$, chloroform- $d) ; \delta 5.08(1 \mathrm{H}, \mathrm{t}, J=6.0,5.4 \mathrm{~Hz}), 5.05(1 \mathrm{H}$, $\mathrm{t}, J=7.2,6.6 \mathrm{~Hz}), 3.27(1 \mathrm{H}, \mathrm{d}, J=10.2 \mathrm{~Hz}), 1.61(3 \mathrm{H}, \mathrm{s}), 1.47(3 \mathrm{H}, \mathrm{s}), 1.03(3 \mathrm{H}, \mathrm{s}), 0.88-0.86(6 \mathrm{H}, \mathrm{dd}$, $\mathrm{J}=4.8,1.8 \mathrm{~Hz}) ;{ }^{13} \mathrm{C}-\mathrm{NMR}(150 \mathrm{MHz}$, chloroform-d): $\delta 16.12,17.99,18.05,18.14,20.66,24.83,30.62,30.67$, $32.31,33.66,34.83,36.34,38.60,75.50,84.15,88.53,121.77,125.10,134.17,134.21$; HRMS (ESI+): $m / z$ $307.2623[\mathrm{M}+\mathrm{H}]^{+}$.

Viridiflorol (19): colorless oil; ${ }^{1} \mathrm{H}-\mathrm{NMR}(600 \mathrm{MHz}$, chloroform- $d) ; \delta 1.17(3 \mathrm{H}, \mathrm{s}), 1.04(3 \mathrm{H}, \mathrm{s}), 1.02(3 \mathrm{H}, \mathrm{s})$, $0.98(3 \mathrm{H}, \mathrm{d}, \mathrm{J}=7.2 \mathrm{~Hz}), 0.64(1 \mathrm{H}, \mathrm{m}), 0.14(1 \mathrm{H}, \mathrm{t}, J=9.0 \mathrm{~Hz}) ;{ }^{13} \mathrm{C}-\mathrm{NMR}(150 \mathrm{MHz}$, chloroform-d): $\delta 16.1$, 16.3, 18.4, 18.8, 22.2, 25.7, 28.5, 28.6, 29.0, 32.1, 37.7, 38.4, 39.7, 58.2, 74.6; HRMS (ESI+): m/z 205.1950 [M $\left.-\mathrm{H}_{2} \mathrm{O}+\mathrm{H}\right]^{+}$.

Iso-serratol (20): colorless oil; ${ }^{1} \mathrm{H}-\mathrm{NMR}(600 \mathrm{MHz}$, chloroform- $d): \delta 5.07(1 \mathrm{H}, \mathrm{t}, J=7.2,6.6 \mathrm{~Hz}), 4.96$ $(1 \mathrm{H}, \mathrm{t}, J=6.6 \mathrm{~Hz}), 4.90(1 \mathrm{H}, \mathrm{t}, J=6.6 \mathrm{~Hz}), 1.52(9 \mathrm{H}, \mathrm{brt}), 1.14(6 \mathrm{H}, \mathrm{s}) ;{ }^{13} \mathrm{C}-\mathrm{NMR}(150 \mathrm{MHz}$, chloroform-d $)$ : $\delta 15.2,15.5,15.5,23.9,24.6,27.4,27.6,28.2,28.4,37.7,38.8,39.3,48.4,73.9,124.9,125.7,125.9,132.9$, 133.2, 134.0; HRMS (ESI+): $m / z 489.2496[\mathrm{M}+\mathrm{H}]^{+}$.

Lupenone (21): white amorphous powder; ${ }^{1} \mathrm{H}-\mathrm{NMR}(600 \mathrm{MHz}$, chloroform- $d)$ : $\delta 4.66(1 \mathrm{H}, \mathrm{brs}, \mathrm{H}-29 \mathrm{a})$, 4.54 (1H, brs, H-29b), $2.46(1 \mathrm{H}, \mathrm{m}), 2.37(1 \mathrm{H}, \mathrm{m}), 1.86(2 \mathrm{H}, \mathrm{m}), 1.68(3 \mathrm{H}, \mathrm{s}), 1.07(6 \mathrm{H}, \mathrm{s}), 1.02(3 \mathrm{H}, \mathrm{s}), 0.95$ $(3 \mathrm{H}, \mathrm{s}), 0.93(3 \mathrm{H}, \mathrm{s}), 0.81(3 \mathrm{H}, \mathrm{s}) ;{ }^{13} \mathrm{C}-\mathrm{NMR}(150 \mathrm{MHz}$, chloroform-d): $\delta 14.4,15.9,16.7,18.0,19.6,19.7$, 21.4, 21.4, 25.7, 26.6, 27.6, 29.7, 33.5, 34.4, 35.8, 36.6, 38.4, 39.4, 40.6, 40.7, 42.6, 43.0, 47.4, 48.0, 48.2, 49.8, $54.9,109.3,150.8,217.7$; HRMS (ESI+): $m / z 447.3829[\mathrm{M}+\mathrm{Na}]^{+}$.

\subsection{In Vitro $\alpha$-Glucosidase Inhibition}

$\alpha$-Glucosidase enzyme (E.C. 3.2.1.20) from Saccharomyces cerevisiae were purchased from Sigma-Aldrich (Darmstadt, Hesse, Germany) with product number of G0660-750UN and their inhibition assay was carried out [23] by using $0.1 \mathrm{M}$ phosphate buffer ( $\mathrm{pH}$ 6.8) solution at $37^{\circ} \mathrm{C}$. After enzyme ( 0.2 units $/ \mathrm{mL}$ ) incubation in phosphate buffered saline for $15 \mathrm{~min}$ with different concentrations of tested 
compounds, the $p$-nitrophenyl- $\alpha$-D-glucopyranoside (substrate, $0.7 \mathrm{mM}$ ) was added and the variation in absorbance at $400 \mathrm{~nm}$ was observed for $30 \mathrm{~min}$ using a spectrophotometer ( $\mathrm{xMark}^{\mathrm{TM}}$ Microplate Spectrophotometer, BIO-RAD, Hercules, CA, USA). For the kinetics studies, different concentrations $(0.1,0.2,0.4$ and $0.8 \mathrm{mM})$ of $p$-nitrophenyl- $\alpha$-D-glucopyranoside (substrate) were used. In control the tested compounds were replaced with DMSO- $d_{6}$ (7.5\% final). Acarbose was used as the standard inhibitor. Three time the experiment was repeated having triplicate of each samples. The $\%$ inhibition was calculated by using the following formula:

$$
\% \text { Inhibition }=100-(\mathrm{OD} \text { test well/OD control }) \times 100(\mathrm{OD}=\text { Optical density })
$$

\subsection{Computational Modeling and Molecular Docking}

Molecular Operating Environment [24] was employed for the docking of four active compounds (3-5 and 11). Previously three-dimensional (3D) coordinates of Saccharomyces cerevisiae $\alpha$-glucosidase enzyme was generated by homology modeling $[25,26]$. The primary sequence of $S$. cerevisiae $\alpha$-glucosidase was retrieved from UniProtKB (AC\#P53341). Homology modeling was carried out on Swiss Model server (https://swissmodel.expasy.org/) by using S. cerevisiae isomaltase (PDB code: 3A47, resolution: $1.59 \AA$ and PDB code: $3 \mathrm{AXH}$, resolution: $1.8 \AA$ ) as templates that has $>72 \%$ identity with the target enzyme. The generated model comprises of 579 residues. The catalytic residues were identified by superimposing S. cerevisiae isomaltase structure (PDB code: $3 \mathrm{AXH}$ ) in complex with isomaltose. The stereochemical properties of model were scrutinized by Procheck (http://services.mbi.ucla.edu/PROCHECK/), ERRAT (http://servicesn.mbi.ucla.edu/ERRAT/) and verify3D (http://servicesn.mbi.ucla.edu/Verify3D/). Procheck results showed that $444(86.7 \%)$, $63(12.3 \%), 3(0.6 \%)$ and two $(0.4 \%)$ residues lied in the most favored, additional allowed, generously allowed and disallowed regions, respectively. ERRAT showed 93.52 quality factor and Verify3D depicted that $95.5 \%$ residues showed average 3D-1D score of 0.7 . The model is of good quality. The allosteric sites were identified by literature review [27-30] and MOE Site-Finder. Protonation state of protein was set according to the neutral $\mathrm{pH}$, and partial charges were applied on protein by using AMBER12: EHT force field. Ten water molecules are involved in protein-substrate bridging in the active site; therefore, the coordinates of those water molecules were transferred in the model from template and retained during docking.

Human $\alpha$-glucosidase (PDB ID: 5NN8) [31] structure was taken from Protein Data Bank; all the heteroatoms and water molecules were removed. Protonation state of protein was demonstrated according to the neutral $\mathrm{pH}$. Protein was treated as described above.

The 3D-structures of the active compounds (3-5 and 11) were constructed on MOE, partial charges were applied on each structure and the structures were minimized with AMBER12: EHT force field until the gradient was reached to $0.1 \mathrm{kcal} / \mathrm{mol} / \AA$ A. Docking was carried out by Triangle matcher docking algorithm and London dG scoring function. The compounds were docked on the predicted allosteric sites to scrutinize their binding potential on different sites of $\alpha$-glucosidase. On each site, thirty docked possess of compounds were saved for interaction analysis. After docking, protein-ligand interaction fingerprints (PLIF) were used to calculate the 2D-interactions of compounds with the binding sites.

\section{Results and Discussion}

\subsection{Structural Elucidation of Compound 1}

Compound 1 (Figure 1) was isolated as white amorphous powder having molecular formula of $\mathrm{C}_{32} \mathrm{H}_{52} \mathrm{O}_{2}$ which was further evidenced by HRMS (ESI ${ }^{+}$) which exhibited molecular ion peaks at $\mathrm{m} / \mathrm{z}$ $m / z 469.3292[\mathrm{M}+\mathrm{H}]^{+}$(calculated for $\mathrm{C}_{32} \mathrm{H}_{53} \mathrm{O}_{2}: 469.3280$ ); (7 degree of unsaturation). The Infrared spectrum of 1 showed characteristic absorption bands at 1724 and 1624 attributed to acetate $\left(\mathrm{CH}_{3} \mathrm{CO}\right)$ and double bond $(\mathrm{C}=\mathrm{C})$. The ${ }^{1} \mathrm{H}-\mathrm{NMR}$ spectrum of 1 showed seven tertiary methyls $(\delta \mathrm{c} 28.0,25.7,24.2$, $20.1,18.6,16.6$ and 15.3 each single), one secondary methyl $\left(\delta_{\mathrm{H}} 0.93, \mathrm{~d}, J=6.4 \mathrm{~Hz} ; \delta c 18.6\right)$, one acetate 
methyl $\left(\delta_{\mathrm{H}} 2.02\right.$, s) and a trisubstituted olefinic proton $\left(\delta_{\mathrm{H}} 5.08, \mathrm{t}=7.2,5.4 \mathrm{~Hz}, \mathrm{H}-24\right)$, which are characteristic of tirucullane-type triterpene acetate [14,32]. The ${ }^{1} \mathrm{H}$ NMR spectrum confirmed the presence of acetate group at $\mathrm{C}-3$ and was in $\alpha$-orientation as evidenced by the doublet of doublet (12.0, $4.8 \mathrm{~Hz}$ ) of the $\beta$-oriented proton which appeared at $\delta_{\mathrm{H}} 4.49$, an interpretation and $\beta$-orientation further substantiated by heteronuclear multiple bond correlation (HMBC) correlation between $\mathrm{H}-5\left(\delta_{\mathrm{H}}\right.$ 1.62) and C-3 ( $\delta \mathrm{c} 80.9)$ and nuclear overhauser effect spectroscopy (NEOSY) correlation between H-3 and $\mathrm{CH}_{3}-23$ position. On the other hand, the singlet peak at $\delta 5.08(\mathrm{H}-24)$ correlated with $\mathrm{C}-25(\delta \mathrm{c}$ 131.2), C-23 ( $\delta 24.9), C-27$ ( $\delta \mathrm{c} 25.7)$ in the HMBC spectrum confirm the position of olefinic double bond between C-24 and C-25. NOESY correlations of Me-18 with H-20 further prove the configuration of $\mathrm{C}-20$ to be $\mathrm{S}$ and thus the affiliation of the triterpene to the tirucallane series [33]. The stereochemistry of the compound is also in complete agreement with the published data [33,34] except acetate group at the $\mathrm{C}-3$ position.

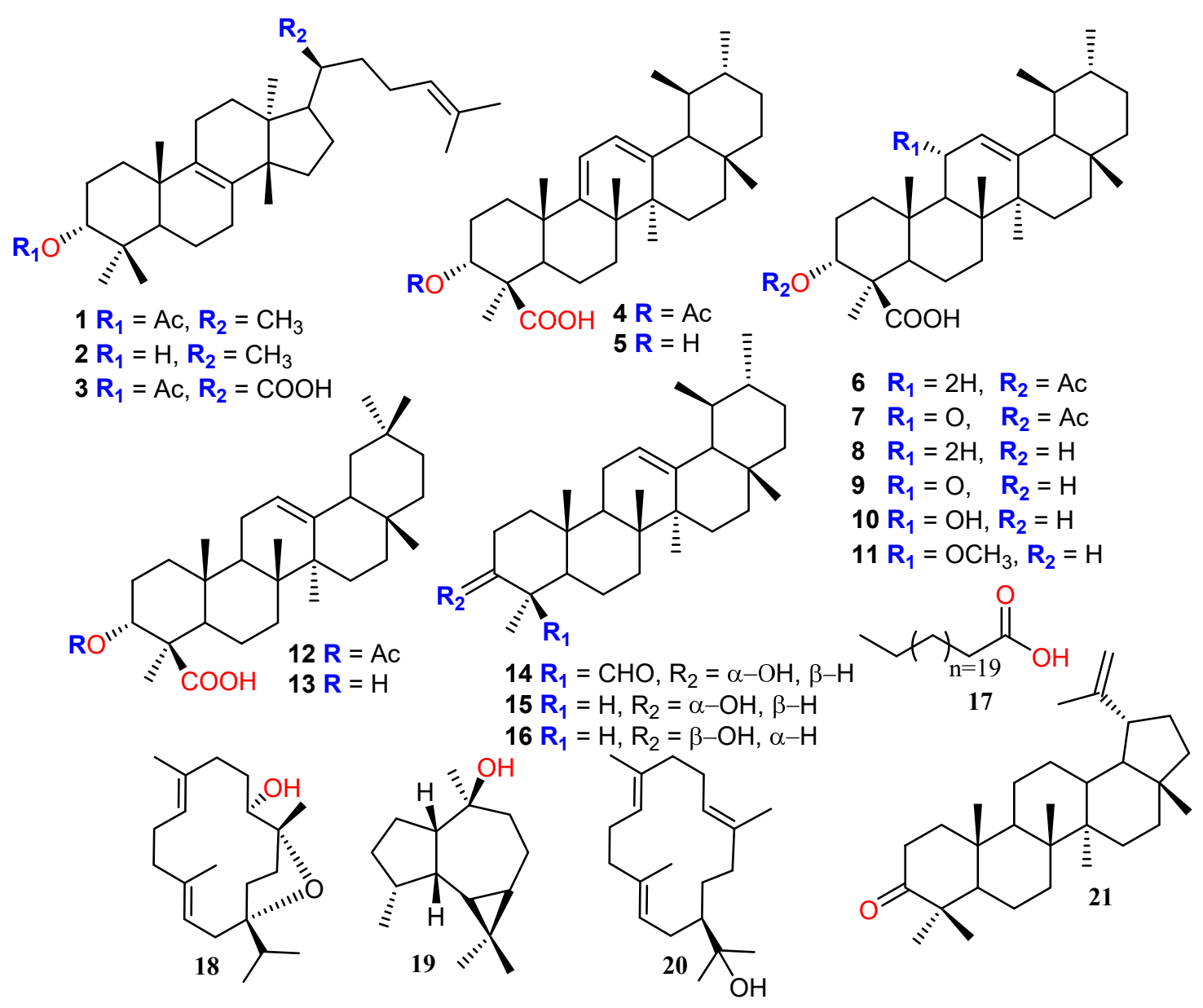

Figure 1. Structures of the compounds 1-21 isolated from B. elongata.

The ${ }^{13} \mathrm{C}$-NMR spectrum of compound 1 displayed 32 peaks accounted for by nine methyls, five methines, ten methylenes and eight quaternary carbons. The ${ }^{13} \mathrm{C}-\mathrm{NMR}$ spectrum of $\mathbf{1}$ also attributed the presence of two olefinic groups at $\delta 133.9$ and 133.6 (C-8 and C-9), 131.2 and 125.2 (C-24 and C-25) and one acetylated carbonyl group at $\delta 171.0$ (C-32). All the positions of the substitutions were deduced using the COSY and HMBC techniques (Figure 2). ${ }^{1} \mathrm{H}$ and ${ }^{13} \mathrm{C}-\mathrm{NMR}$ data were in complete agreement with those published $[33,35,36]$. Compound 1 was thus assigned the structure of $5 \alpha$-tirucalla-8,24-dien-3 $\alpha$-acetate [37] obtained this compound by partial synthesis, while its hydroxyl analogs $5 \alpha$-tirucalla-8,24-dien- $3 \alpha$-ol and $5 \alpha$-tirucalla-7, 24-dien-3 $\beta$-ol were previously published [33,38]. But, to the best knowledge, it has not been described as a natural product before. 


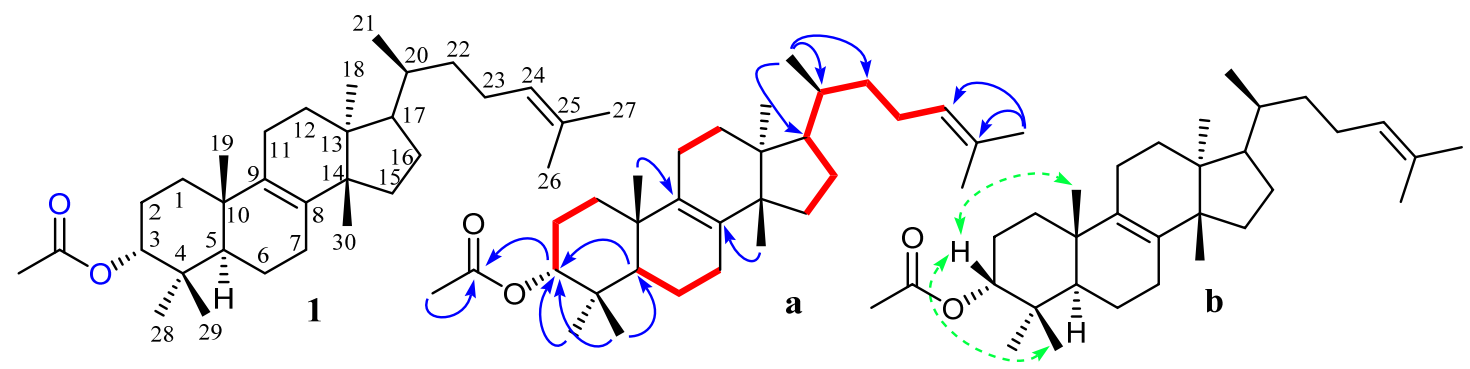

Figure 2. Structure of the compound 1; (a) key heteronuclear multiple bond correlation HMBC (blue arrow) and H-H COSY (red line) correlations; (b) key nuclear overhauser effect spectroscopy NOESY correlations of compound 1 .

The structures of the known compounds including $5 \alpha$-tirucalla-8,24-dien-3 $\alpha$-ol (2) [33] $3 \alpha$-acetoxytirucall-8,24-dien-21-oic acid (3) [32,39] 3-O-acetyl-9,11-dehydro- $\beta$-boswellic acid (4), 9,11-dehydro- $\beta$-boswellic acid (5) [32,40,41] 3-acetyl- $\beta$-boswellic acid ( $\beta$-ABA, 6), 3-acetyl 11-keto- $\beta$-boswellic acid (AKBA, 7), $\beta$-boswellic acid ( $\beta$-BA, 8), 11-keto- $\beta$-boswellic acid (KBA, 9), $3 \alpha, 11 \alpha$-dihydroxyurs-12-en-24-oic acid (10) [11,41] $11 \alpha$-methoxy- $\beta$-boswellic acid (11) [42] 3 -acetyl- $\alpha$-boswellic acid ( $\alpha$-ABA, 12), $\alpha$-boswellic acid $(\alpha$-BA, 13), $\beta$-boswellic aldehyde (14), epi- $\alpha$-amyrin (15), $\alpha$-amyrin (16) [43] tricosanoic acid (17) [44], incensole (18) [32,45], viridiflorol (19) [46], iso-serratol (20) $[47,48]$ and lupenone $(\mathbf{2 1})[49,50]$ were determined on the basis of spectroscopic techniques and by comparison with the published data (Figure 1).

\section{2. $\alpha$-Glucosidase Inhibition and Structural-Activity Relationship (SAR)}

All the isolated compounds 1-21 were screened for $\alpha$-glucosidase enzyme inhibition at $1.0 \mathrm{mM}$ concentration (Table 1 ). In the preliminary screening, four compounds (3-5 and 11) demonstrated significant in vitro $\alpha$-glucosidase inhibitory properties with $\mathrm{IC}_{50}$ values in the range of $9.9 \pm 0.48-56.8 \pm 1.30 \mu \mathrm{M}$, while compounds $\mathbf{1}, \mathbf{2}, \mathbf{1 9}$ and 20 displayed \% inhibition less than 50, therefore, were not evaluated for $\mathrm{IC}_{50}$. The remaining isolated compounds belonging to different classes including diterpenoids, triterpenoids and boswellic acids were already reported by our group with SAR study [11].

Table 1. $\alpha$-Glucosidase inhibition of active constituents from B. elongata.

\begin{tabular}{cccc}
\hline Code & $\mathbf{I C}_{\mathbf{5 0}}=\boldsymbol{\mu M} \pm \mathbf{S E M}$ & $\mathbf{K} \boldsymbol{i}=\boldsymbol{\mu M} \pm \mathbf{S E M}$ & Type of Inhibition \\
\hline $\mathbf{1}$ & $\mathrm{NA}$ & $\mathrm{ND}$ & ND \\
$\mathbf{2}$ & $\mathrm{NA}$ & $\mathrm{ND}$ & ND \\
$\mathbf{3}$ & $56.8 \pm 1.30$ & $51.15 \pm 0.63$ & Non-competitive \\
$\mathbf{4}$ & $9.9 \pm 0.48$ & $7.05 \pm 0.75$ & Non-competitive \\
$\mathbf{5}$ & $20.9 \pm 0.05$ & $15.30 \pm 0.54$ & Non-competitive \\
$\mathbf{1 1}$ & $14.9 \pm 1.31$ & $8.05 \pm 0.38$ & Non-competitive \\
$\mathbf{1 9}$ & $\mathrm{NA}$ & $\mathrm{ND}$ & ND \\
$\mathbf{2 0}$ & $\mathrm{NA}$ & $\mathrm{ND}$ & ND \\
Acarbose & $942 \pm 0.74$ & - & - \\
\hline
\end{tabular}

$\mathrm{NA}=$ Not active; SEM = Standard error Mean; ND = Not determined.

Comparing boswellic acids, compound $4(9.9 \pm 0.48 \mu \mathrm{M})$ showed potent inhibition followed by $11(14.9 \pm 1.31 \mu \mathrm{M})$ and $5(20.9 \pm 0.05 \mu \mathrm{M})$. Compound 4 exhibited highest inhibition against $\alpha$-glucosidase enzyme compared all types of other boswellic acids reported in the literature until now [11,51]. Higher inhibition of 4 compared to 5 (both have same basic structure) may be due to the replacement of $-\mathrm{OH}$ with acetyl group resulted in the increase of $\alpha$-glucosidase activity. The compound 4 was found to be 94 times more active than the clinically standard inhibitor acarbose $\left(\mathrm{IC}_{50}=942 \pm 0.74 \mu \mathrm{M}\right)$. The previous investigation showed that $\alpha$-ABA, $\beta$-ABA and AKBA were the most promising glucosidase inhibitors having acetyl group at $\mathrm{C}-3$ position. 
Compound 3, carrying $\mathrm{COOH}$ group, exhibited higher activity $\left(\mathrm{IC}_{50}=56.8 \pm 1.30 \mu \mathrm{M}\right)$ than 1 (inactive) having methyl group at C-20, indicating that the higher activity of compound 3 may be due the replacement of methyl group with carboxylic acid. Similarly, comparing compound 11 with 10, the higher activity of $\mathbf{1 1}$ may be due to the replacement of hydroxyl group with $-\mathrm{OCH}_{3}$ at $\mathrm{C}-11$ position, while the remaining skeleton of both compounds is same. From SAR perspective, among all samples tested for the inhibition of $\alpha$-glucosidase, we conclude that the presence of acetyl group at the C-3 $\alpha$ position and carboxylic acid at C-24 position in the ursane type boswellic acids is essential.

\subsection{Kinetics Studies}

To investigate the mode of interaction and dissociation constant of these potent compounds, the kinetics studies on active compounds $\mathbf{3} \mathbf{- 5}$ and $\mathbf{1 1}$ were performed, with different concentrations of test compounds and substrates. These compounds inhibited the $\alpha$-glucosidase enzyme in a concentration-dependent manner with $K i$ values were between $7.05 \pm 0.75-51.15 \pm 0.63 \mu \mathrm{M}$. From the kinetics studies, it was deduced that the compounds $\mathbf{3 - 5}$ and $\mathbf{1 1}$ are non-competitive inhibitors with $K_{i}$ values in range $77.05 \pm 0.75-51.15 \pm 0.63 \mu \mathrm{M}$. The type of inhibition was determined by Lineweaver-Burk plots, the reciprocal of the rate of the reaction was plotted against the reciprocal of substrate concentrations to monitor the effect of inhibitor on both $K_{m}$ and $V_{\max }$. It was observed from Lineweaver-Burk plots that all compounds 3-5 and $\mathbf{1 1}$ clearly showed non-competitive inhibition Figures 3, 4, 5 and $6 \mathrm{~A}$. In non-competitive inhibition, the $V_{\max }$ of enzyme decreased, while $K_{m}$ are not affected. The Lineweaver-Burk plots (section A) of all Figures 3-6 showed that in the presence of compounds 3-5 and 11 the $V_{\max }$ of $\alpha$-glucosidase enzyme decreased significantly, while the $K_{m}$ remain constant, which indicated the mixed-type of inhibition. The secondary replots of Lineweaver-Burk plots were used to determine the $K_{i}$ values. The Ki values were calculated by plotting the slope of each line in the Lineweaver-Burk plots against different concentrations of compounds 3-5 and 11 (Figures 3, $4,5$ and $6 \mathrm{~B})$. The $K_{i}$ value was confirmed from Dixon plot by plotting the reciprocal of the rate of reaction against different concentrations of compounds 3-5 and $\mathbf{1 1}$ (Figures 3, 4, 5 and 6C).

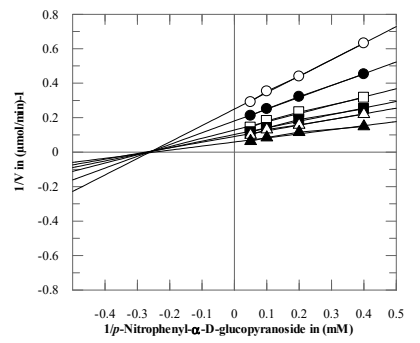

(A)

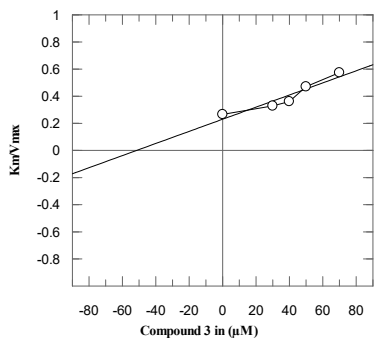

(B)

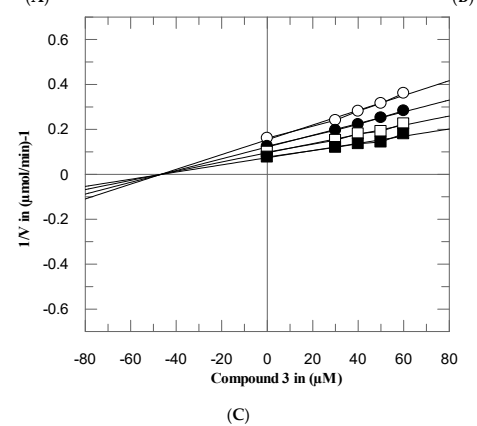

Figure 3. The inhibition of $\alpha$-glucosidase by compound 3 (A) Lineweaver-Burk plot of reciprocal of rate of reaction (velocities) vs. reciprocal of substrate ( $p$-nitrophenyl- $\alpha$-D-glucopyranoside) in the absence $(\Delta)$, and in presence of $30(\Delta), 40(\mathbf{\square}), 50(\square), 60(\bullet)$, and $70 \mu \mathrm{M}(\bigcirc)$ of compound 3. (B) Secondary replot of Lineweaver-Burk plot between the slopes of each line on Lineweaver-Burk plot vs. different concentrations of compound 3. (C) Dixon plot of reciprocal of rate of reaction (velocities) vs. different concentrations of compound 3. 

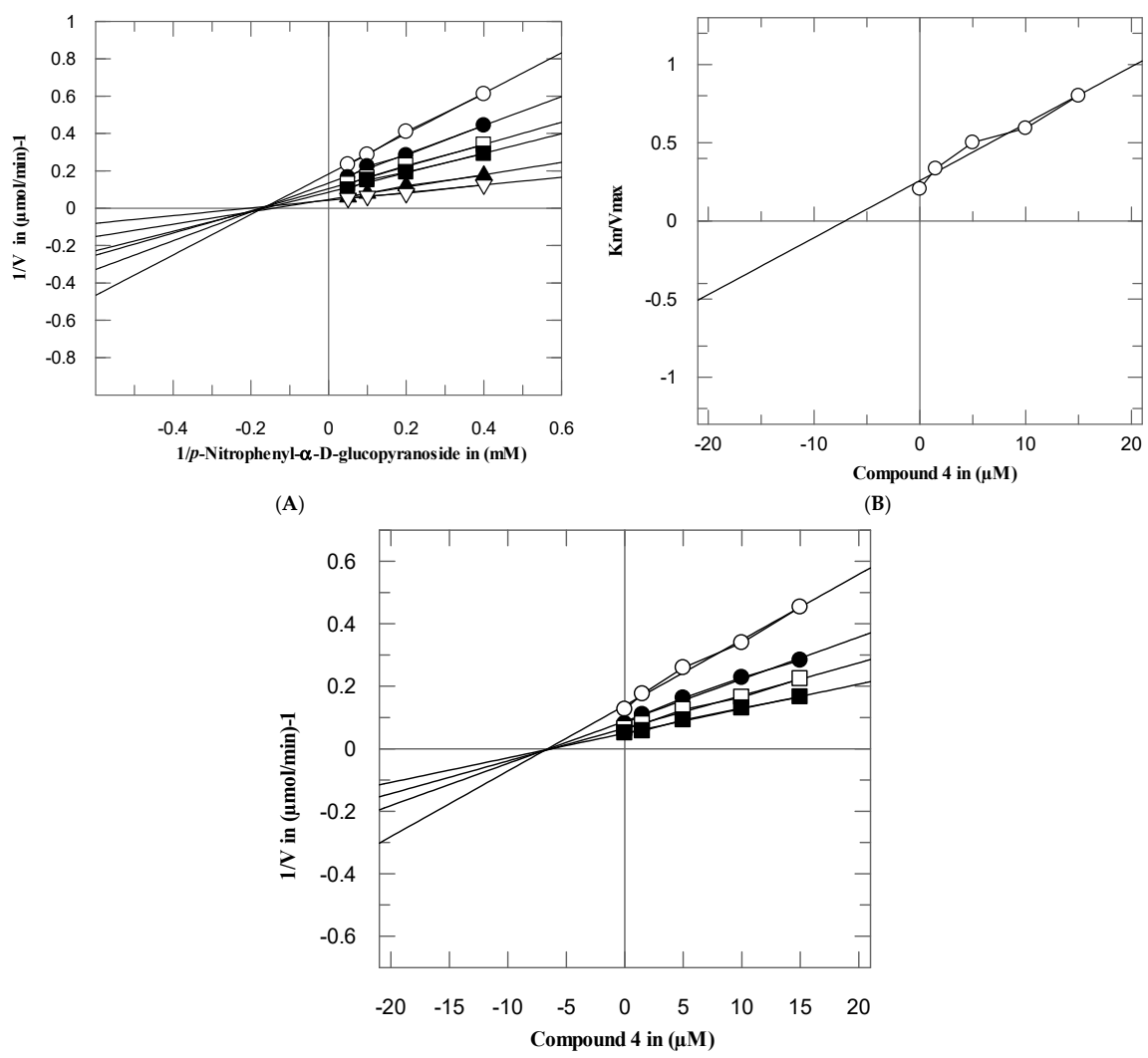

(B)

(C)

Figure 4. The inhibition of $\alpha$-glucosidase by compound 4 (A) Lineweaver-Burk plot of reciprocal of rate of reaction (velocities) vs. reciprocal of substrate ( $p$-nitrophenyl- $\alpha$-D-glucopyranoside) in the absence $(\Delta)$, and in presence of $2.5(\mathbf{\Lambda}), 5(\square), 10(\square), 15(\bullet)$, and $20 \mu \mathrm{M}(\bigcirc)$ of compound 4 . (B) Secondary replot of Lineweaver-Burk plot between the slopes of each line on Lineweaver-Burk plot vs. different concentrations of compound 4. (C) Dixon plot of reciprocal of rate of reaction (velocities) vs. different concentrations of compound 4 .

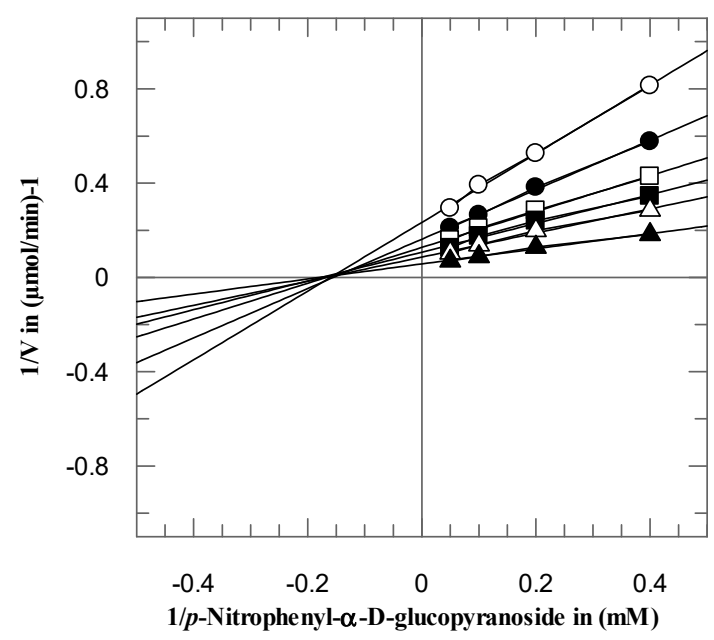

(A)

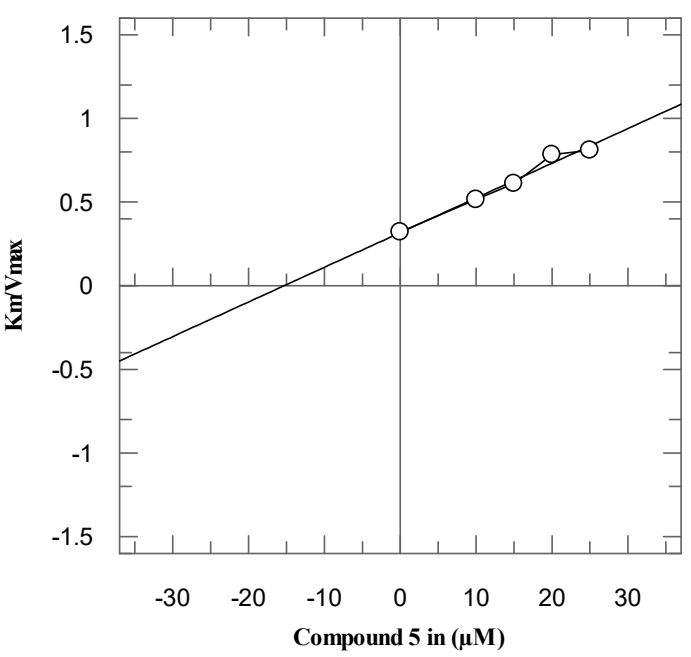

(B)

Figure 5. Cont. 


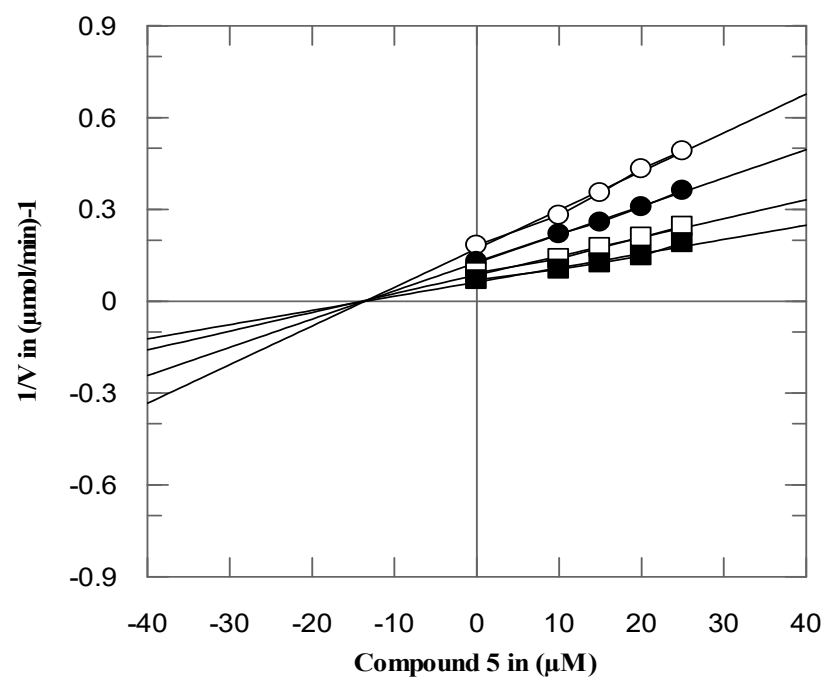

(C)

Figure 5. The inhibition of $\alpha$-glucosidase by compound 5 (A) Lineweaver-Burk plot of reciprocal of rate of reaction (velocities) vs. reciprocal of substrate ( $p$-nitrophenyl- $\alpha$-D-glucopyranoside) in the absence $(\boldsymbol{\Lambda})$, and in presence of $10(\Delta), 15(\square), 20(\square), 20(\bullet)$, and $25 \mu \mathrm{M}(\bigcirc)$ of compound 5. (B) Secondary replot of Lineweaver-Burk plot between the slopes of each line on Lineweaver-Burk plot vs. different concentrations of compound 5. (C) Dixon plot of reciprocal of rate of reaction (velocities) vs. different concentrations of compound 5 .

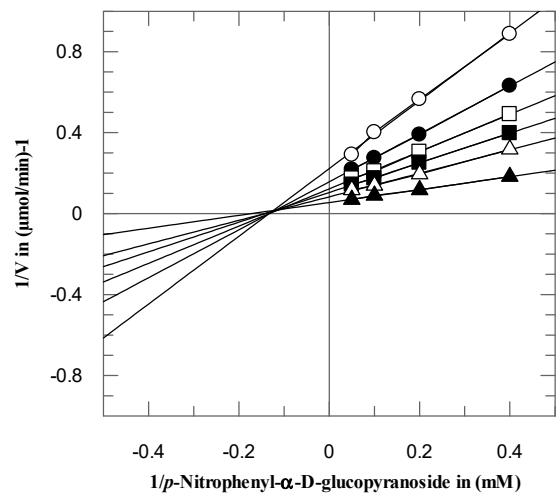

(A)

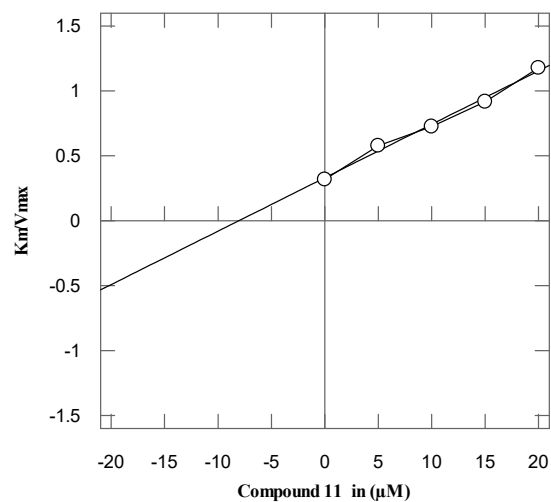

(B)

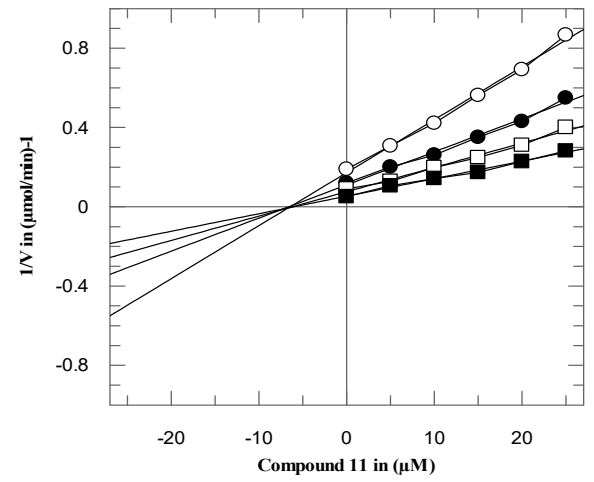

(C)

Figure 6. The inhibition of $\alpha$-glucosidase by compound 11 (A) Lineweaver-Burk plot of reciprocal of rate of reaction (velocities) vs. reciprocal of substrate ( $p$-nitrophenyl- $\alpha$-D-glucopyranoside) in the absence $(\boldsymbol{\Delta})$, and in presence of $5(\Delta), 10(\boldsymbol{\square}), 15(\square), 20(\bullet)$, and $25 \mu \mathrm{M}(\bigcirc)$ of compound 11. (B) Secondary replot of Lineweaver-Burk plot between the slopes of each line on Lineweaver-Burk plot vs. different concentrations of compound 11. (C) Dixon plot of reciprocal of rate of reaction (velocities) vs. different concentrations of compound $\mathbf{1 1}$. 


\subsection{Molecular Docking of $\alpha$-Glucosidase Inhibitors}

The isolated compounds (3-5 and 11) exhibited significant non-competitive inhibition of $\alpha$-glucosidase in vitro. These triterpenic acids are involved in the allosteric modulation of $\alpha$-glucosidase. Therefore, we identified several hotspots as allosteric sites (Table 2) of $\alpha$-glucosidase and performed molecular docking to predict the mode of binding of compounds in the predicted allosteric sites of $S$. cerevisiae $\alpha$-glucosidase. Previously 3D-coordinates of $S$. cerevisiae $\alpha$-glucosidase were generated by homology modeling to be used in molecular docking studies. The active site of the enzyme comprises of a catalytic triad (Asp214, Glu276 and Asp349) where Asp214 work as nucleophile, Glu276 act as a proton donor for substrate, and the transition state of substrate is stabilized by Asp349. Additionally, several residues (Asp68, Tyr71, Val108, His111, Phe157, Phe158, Phe177, Gln181, Arg212, Thr215, Leu218, Glu276, Ala278, Phe300, Arg312, His348, Asp349, Gln350, Asp408, Arg439 and Arg443) creates the lining of active site and provide strong hydrophilic and hydrophobic interactions to the substrate molecule. These residues also stabilize the inhibitor acarbose. In the active site of $\alpha$-glucosidase many water molecules (Wat1021, Wat1026, Wat1056, Wat1058, Wat1061, Wat1087, Wat1102, Wat1122, Wat1174 and Wat1228) are involved in enzyme-substrate and enzyme-inhibitor bridging. The rim of the active site gorge is surrounded by the gate keeping residues (Phe231, His239, Asn241, His279, Glu304, Arg312) that regulate the entry and exit of ligand in the active site. The enzyme substrate complex is shown in Figure 7.

Table 2. Predicted allosteric sites of S. cerevisiae $\alpha$-glucosidase enzyme.

\begin{tabular}{ccc}
\hline Predicted Allosteric Sites & Binding Residues & References \\
\hline 1 & $\begin{array}{c}\text { Lys12, Trp14, His258, Lys262, Val265, } \\
\text { Glu270, Ile271, Thr287, Ala289, Tyr292, } \\
\text { Val294, Thr295, Ser339 }\end{array}$ & {$[27,28]$} \\
\hline 2 & $\begin{array}{c}\text { Thr287, Val297, Ser299, His302, Ile334, } \\
\text { Trp340, Ala341, Thr342, Tyr344 }\end{array}$ & {$[29]$} \\
\hline 3 & $\begin{array}{c}\text { Thr9, Pro11, Lys15, Ile334, Asn335, Ser339, } \\
\text { Trp340, Thr380, Tyr508, Tyr510, Tyr529 }\end{array}$ & {$[28]$} \\
\hline 4 & $\begin{array}{c}\text { Gln66, Gln67, Met69, Ser179, Arg180, } \\
\text { Glu405, Val407, Lys410, Asn411, Trp465 }\end{array}$ & {$[27]$} \\
\hline 5 & $\begin{array}{c}\text { Tyr142, Ile149, Lys147, Pro150, Asp227, } \\
\text { Asp232, Ile236 }\end{array}$ & {$[28]$} \\
\hline & Lys155, Phe157, Leu176, Leu237, Gln238, \\
Gly243, Ser244, Phe311, Arg312 & {$[28]$} \\
\hline
\end{tabular}

In order to determine the non-competitive behavior of compounds $\mathbf{3}-\mathbf{5}$ and 11, different allosteric sites were recognized by literature review that reveals six potential hotspots are present as allosteric sites in S. $\alpha$-glucosidase enzyme (Table 2). [27] revealed that two non-competitive inhibitors (oleanolic acid and ursolic acid) binds at two different sites to induce allosteric regulation. It was shown that oleanolic acid binds at allosteric site (AS)-1 which is created by Trp14, Lys12, Ser295, Ala289, His258, Tyr292, Lys262, Val265, Ile271 and Glu270 while ursolic acid binds at AS-4 (Gln66, Gln67, Met69, Ser179, Arg180, Glu405, Val407, Lys410, Asn411, Trp465) [27] demonstrated that a mixed type inhibitor ((E)-3-butylideneisobenzofuran-1(3H)-one) binds to a site close to the catalytic site and is formed by residues Thr287, Val297, Ser299, His302, Ile334, Trp340, Ala341, Thr342 and Tyr344. This site was considered as AS-2 in the current docking studies. Moreover, AS-1, AS-3, AS-5 and AS-6 were identified by [30] as the binding site for some xanthone derivatives that exhibited non-competitive inhibition of $\alpha$-glucosidase. AS-1 to AS-3 is located away from the active site, while AS-4 to AS-6 is situated near the active site. The compounds 3-5 and $\mathbf{1 1}$ were targeted at all the sites (AS-1 to AS-6) individually and the docked conformation of each molecule with the high negative docking score was considered as the most optimal binding orientation and selected for binding mode analysis. 
The optimal conformations of 3-5 and $\mathbf{1 1}$ were well accommodated inside AS-1 and AS-4 where compound 4 exhibited highest binding potential (>-10), followed by compounds $11(->9), 5(-9)$ and $3(>-8)$. The docking scores of compounds at AS-2, were in range of -4 to $>-6$, suggesting that this may not be an appropriate binding site for our compounds (supporting information, Table S1). Similarly, compounds did not possess good binding potential for AS-3 and exhibited docking scores in range of $>-6$ to $>-7$ and the docked conformations of compounds were surface exposed that did not show favorable binding interactions. However, compounds demonstrated good binding potential for AS-5 (docking scores in range of $>-8$ to $>-7$ ) compared to AS-2 and AS-3, however lower score than AS-1. When docked at AS-6, all the compounds remained surface exposed therefore displayed least binding potential ( $>-6$ to $>-7$ ), thus it was considered as the most unappropriated binding site for these triterpenic acids. The docking results are summarized in Table S1, in supporting information.

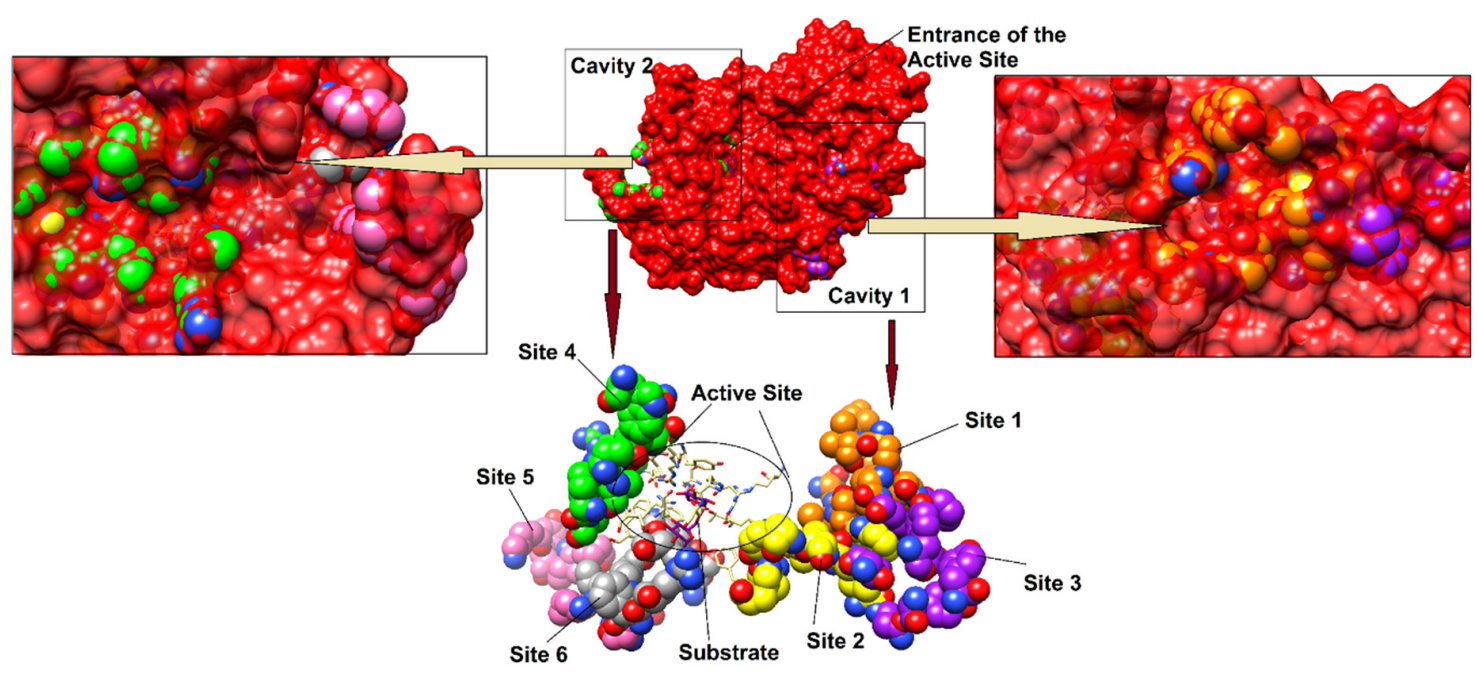

Figure 7. Structural topology of $S$. cerevisiae $\alpha$-glucosidase is shown. The generated cavities are displayed in boxes. The predicted allosteric sites are shown in sphere model, each site is labeled. The active site residues (shown in yellow sticks) are presented in complex with the substrate molecule (isomaltose, shown in purple sticks).

AS-1 to AS-3 are located opposite to the active site, we selected AS-1 to AS-3 communally (named as Cavity 1) and docked the compounds at this cavity. Based on docking scores and binding interactions, the compounds depicted significantly higher binding potential at this cavity. The most active compound (4) showed -10.77 docking score, followed by compounds $\mathbf{1 1}(-10.69), \mathbf{5}(-10.21)$ and 3 (-9.83). Additionally, Cavity 2 was created by combining AS-4 to AS-6 (Gln66, Gln67, Met69, Tyr142, Lys147, Ile149, Pro150, Lys155, Phe157, Leu176, Ser179, Arg180, Asp227, Asp232, Ile236, Leu237, Gln238, Gly243, Ser244, Phe311, Arg312, Glu405, Val407, Lys410, Asn411, Trp465). The compounds exhibited $>-10$ to $>-8$ docking score at cavity 2 . The docking scores indicate that compounds have higher binding potential for cavity 1 than cavity 2 . The binding orientation also showed that compounds are well accommodated at the groove present in the cavity 1 . All the predicted sites, and cavities $\mathbf{1}$ and $\mathbf{2}$ are shown in Figure 7. The compounds 4, $\mathbf{5}$ and $\mathbf{1 1}$ are lodged at AS-1 in the cavity 1 and stabilized by hydrogen bonding with Ile271 and His258. The carboxylic group of $\mathbf{4}$ and $\mathbf{1 1}$ accepts H-bond from the amino nitrogen of Ile271, while the -OH of compound 5 donates $\mathrm{H}$-bond to the carbonyl oxygen of His258. The docking mode of $\mathbf{3}$ depict that compound is located at AS-1, however the acetate group of 3 interact with the side chain of Lys15 of AS-3. The docking scores of compounds at cavity 1 and their binding interactions are tabulated in Table 3. The docked conformations of compounds in cavity 1 are presented in Figure 8. The docking score are well correlated with the in vitro experimental findings. 
Table 3. Docking scores and binding interactions of compounds $\mathbf{3} \mathbf{- 5}$ and $\mathbf{1 1}$ at Cavities $\mathbf{1}$ and $\mathbf{2}$ of $S$. Cerevisiae $\alpha$-Glucosidase and Human $\alpha$-Glucosidase.

\begin{tabular}{|c|c|c|c|c|c|}
\hline \multirow{4}{*}{ Compounds } & \multicolumn{5}{|c|}{ Saccharomyces Cerevisiae $\alpha$-Glucosidase } \\
\hline & \multicolumn{5}{|c|}{ Cavity 1} \\
\hline & \multirow{2}{*}{ Scores } & \multicolumn{4}{|c|}{ Binding Interactions } \\
\hline & & Ligand & Receptor & Interaction & Distance (Å) \\
\hline 3 & -9.83 & O77 & NZ-LYS15 & HBA & 3.21 \\
\hline 4 & -10.77 & O75 & N-ILE271 & HBA & 3.00 \\
\hline 5 & -10.21 & $\mathrm{O} 70$ & O-HIS258 & HBA & 3.26 \\
\hline \multirow[t]{2}{*}{11} & -10.69 & $\mathrm{O} 78$ & N-ILE271 & HBA & 2.85 \\
\hline & \multicolumn{5}{|c|}{ Cavity 2} \\
\hline \multirow{2}{*}{ Compounds } & \multirow{2}{*}{ Scores } & \multicolumn{4}{|c|}{ Binding Interactions } \\
\hline & & Ligand & Receptor & Interaction & Distance $(\AA ̊)$ \\
\hline 3 & -8.71 & O84 & NZ-LYS418 & HBA & 2.72 \\
\hline 4 & -10.73 & O79 & N-SER179 & HBA & 3.01 \\
\hline 5 & -9.60 & O77 & ND2-ASN411 & HBA & 2.58 \\
\hline 11 & -9.98 & O81 & N-SER179 & HBA & 2.97 \\
\hline \multicolumn{6}{|c|}{ Human $\alpha$-Glucosidase } \\
\hline & & \multicolumn{4}{|c|}{ Binding Interactions } \\
\hline Compounds & Scores & Ligand & Receptor & Interaction & Distance (̊̊) \\
\hline \multirow{2}{*}{ 3-17 } & \multirow{2}{*}{-9.02} & O77 & N-GLU869 & HBA & 2.78 \\
\hline & & O84 & NE-ARG585 & HBA & 3.39 \\
\hline \multirow{2}{*}{$4-39$} & \multirow{2}{*}{-10.59} & $\mathrm{O} 75$ & NE-ARG585 & HBA & 2.89 \\
\hline & & $\mathrm{O} 75$ & NH2-ARG585 & HBA & 3.32 \\
\hline \multirow{2}{*}{$5-63$} & \multirow{2}{*}{-9.83} & $\mathrm{O} 70$ & NH2-ARG585 & HBA & 1.93 \\
\hline & & C58 & 5-ring-HIS584 & $\mathrm{H}-\pi$ & 3.99 \\
\hline \multirow{2}{*}{$11-92$} & \multirow{2}{*}{-10.07} & O71 & NH2-ARG585 & HBA & 2.99 \\
\hline & & O81 & NH2-ARG608 & HBA & 3.19 \\
\hline
\end{tabular}

HBA = Hydrogen Bond Acceptor.
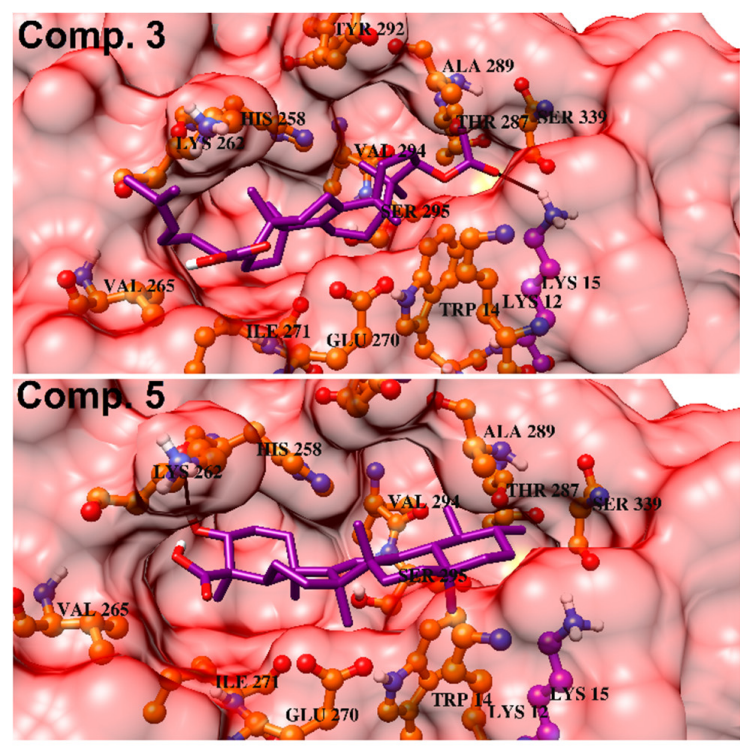

Figure 8. Docked orientations of compounds $\mathbf{3}-\mathbf{5}$ and $\mathbf{1 1}$ are shown in the Cavity 1 . The compounds are shown in purple sticks, hydrogen bonds are presented in black lines.
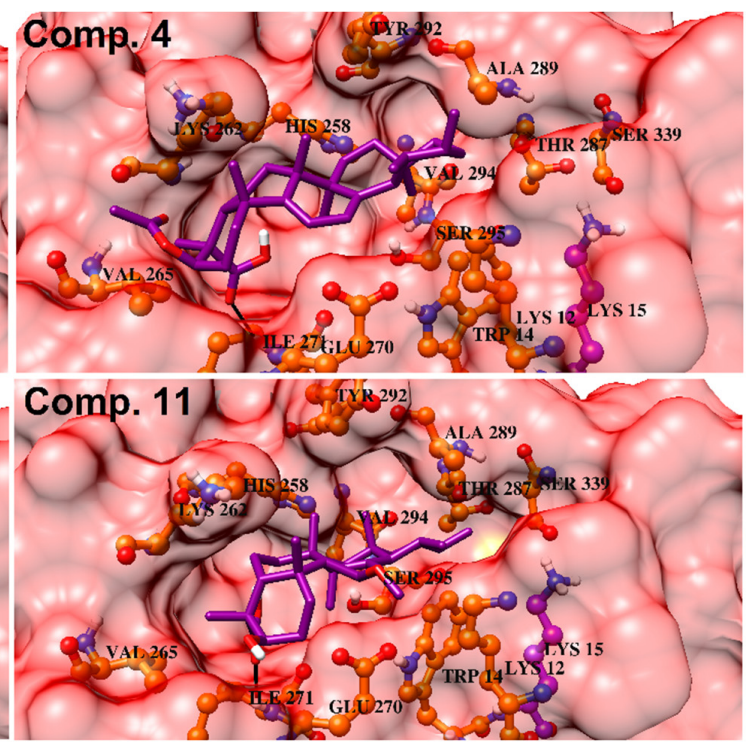
Additionally, compounds 3-5 and $\mathbf{1 1}$ were docked at the allosteric binding site of Human $\alpha$-glucosidase [31,52]. The compounds showed excellent binding affinities and interactions. The acetic and the enoic acid moieties of compound 3 mediate H-bonding with the side chains of Glu896 and Arg585. Similarly, the carboxylic acid moiety of Compound 4 mediated bidentate interactions with the side chain of Arg585. The -OH moiety of compound 5 interact with the side chain of Arg585. Moreover, His584 provide $\mathrm{H}-\pi$ interaction to the compound. The -OH and the methoxy groups of compound 11 interact with the side chains of Arg585 and Arg608, respectively. The docking interactions suggest that the compound has binding potential with the human $\alpha$-glucosidase as well. This is also confirmed by the docking score (Table 3). The binding mode of compounds is shown in Figure 9.
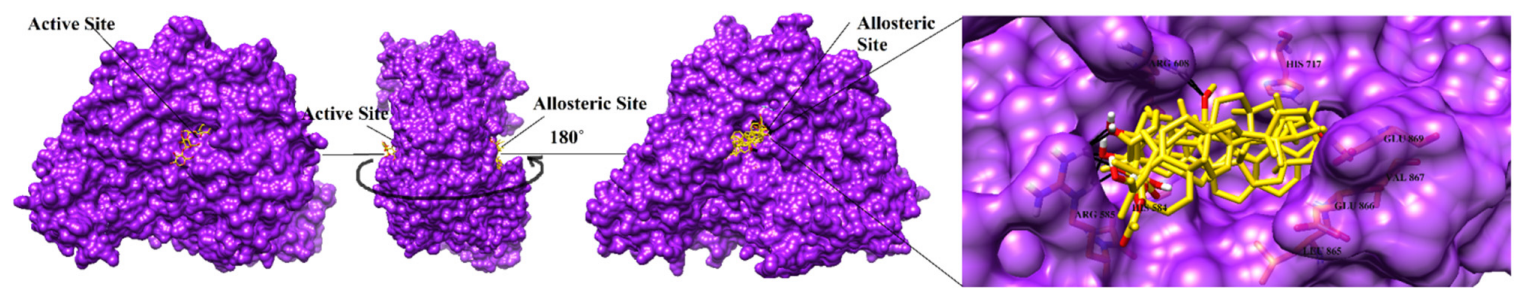

Figure 9. Three-dimensional structure of Human $\alpha$-glucosidase. The active site and the allosteric binding sites are also demonstrated. The binding mode of acarbose (yellow stick model) is shown in the active site of human $\alpha$-glucosidase. The binding mode of compounds 3-5 and $\mathbf{1 1}$ (shown in yellow stick model) in the allosteric site of Human $\alpha$-glucosidase is highlighted. The interacting residues are shown in orange sticks while hydrogen bonds are displayed in black lines.

Moreover, absorption, distribution, metabolism, excretion and toxicity (ADMET) of compounds were scrutinized by admetSAR (http://mmd.ecust.edu.cn/admetsar2/). The results indicate that compounds are non-mutagenic and non-carcinogenic. Caco-2 cells are a human colon epithelial cancer cell line used as a model of human intestinal absorption of drugs and other compounds. It was observed that compounds 3, $\mathbf{4}$ and $\mathbf{1 1}$ are Caco-2 negative means impermeable, while compound $\mathbf{4}$ is Caco-2 positive, suggesting that 4 is permeable to human intestinal cell. Moreover, compounds 2, 5 and $\mathbf{1 1}$ and non-permeable to blood brain barrier, and none of the compound displayed cytochrome inhibitory promiscuity. The gastrointestinal absorption of compounds 3-5 is low, while $\mathbf{1 1}$ has high GI absorption, thus it is demonstrated that compound $\mathbf{1 1}$ would be as excellent drug like molecule because of its tendency to pass through GIT. All the compounds (3-5 and 11) are not substrate of P-glycoprotein, however, compounds 3 and 4 may serve as inhibitor of P-glycoprotein. The calculated acute oral toxicity of compounds $\mathbf{3}-\mathbf{5}$ and $\mathbf{1 1}$ are 1.641, 2.381, 2.469 and $3.201 \mathrm{~kg} / \mathrm{mol}$, respectively. It indicates that compounds 3 and 4 belong to category III, while compounds 5 and $\mathbf{1 1}$ are from category I. The category I comprises of compounds with $\mathrm{LD}_{50}$ values $\leq 50 \mathrm{mg} / \mathrm{kg}$, category II possesses compounds with $\mathrm{LD}_{50}$ values $\geq 50 \mathrm{mg} / \mathrm{kg}$ but $\leq 500 \mathrm{mg} / \mathrm{kg}$, category III includes compounds with $L_{50}$ values $\geq 500 \mathrm{mg} / \mathrm{kg}$ but $\leq 5000 \mathrm{mg} / \mathrm{kg}$ and category IV consisted of compounds with $\mathrm{LD}_{50}$ values $\geq 5000 \mathrm{mg} / \mathrm{kg}$. The results showed that compounds 3 and 4 are safer that compounds 5 and 11. The Human oral bioavailability Score of all the compounds is 0.56 , indicating moderate bioavailability. The results are tabularized in Table 4.

Table 4. Absorption, distribution, metabolism, excretion and toxicity (ADEMT) properties of Compounds $\mathbf{3} \mathbf{- 5}$ and $\mathbf{1 1 .}$

\begin{tabular}{|c|c|c|c|c|c|}
\hline \multirow{2}{*}{ S\# } & \multirow{2}{*}{ Properties } & \multicolumn{4}{|c|}{ Compounds } \\
\hline & & 3 & 4 & 5 & 11 \\
\hline 1 & Ames mutagenesis & - & - & - & - \\
\hline 2 & Acute Oral Toxicity & III & III & I & I \\
\hline 3 & Blood Brain Barrier & - & + & - & - \\
\hline 4 & Caco-2 & - & - & + & - \\
\hline
\end{tabular}


Table 4. Cont.

\begin{tabular}{|c|c|c|c|c|c|}
\hline \multirow{2}{*}{ S\# } & \multirow{2}{*}{ Properties } & \multicolumn{4}{|c|}{ Compounds } \\
\hline & & 3 & 4 & 5 & 11 \\
\hline 5 & Carcinogenicity & - & - & - & - \\
\hline 6 & CYP1A2 inhibition & - & + & - & - \\
\hline 7 & CYP2C19 inhibition & - & - & - & - \\
\hline 8 & CYP2C9 inhibition & - & - & - & - \\
\hline 9 & CYP2C9 substrate & - & - & - & - \\
\hline 10 & CYP2D6 inhibition & - & - & - & - \\
\hline 11 & CYP2D6 substrate & - & - & - & - \\
\hline 12 & CYP3A4 inhibition & - & - & - & - \\
\hline 13 & CYP3A4 substrate & + & + & + & + \\
\hline 14 & CYP inhibitory promiscuity & - & - & - & - \\
\hline 15 & Human Intestinal Absorption & low & low & low & high \\
\hline 16 & Human oral bioavailability Score & 0.56 & 0.56 & 0.56 & 0.56 \\
\hline 17 & Acute Oral Toxicity & $1.641 \mathrm{~kg} / \mathrm{mol}$ & $2.381 \mathrm{~kg} / \mathrm{mol}$ & $2.469 \mathrm{~kg} / \mathrm{mol}$ & $3.201 \mathrm{~kg} / \mathrm{mol}$ \\
\hline 18 & P-glycoprotein inhibitor & + & + & - & - \\
\hline 19 & P-glycoprotein substrate & - & - & - & - \\
\hline 20 & Water solubility & -4.92347 & -4.67124 & -3.78695 & -3.90081 \\
\hline
\end{tabular}

\section{Conclusions}

One new triterpene 1 together with twenty known compounds (2-21) were isolated, first time, from the methanolic extract of the oleo-gum resin of B. elongata. Eight compounds (1-5, 11, 19 and 20) were further screened for in vitro $\alpha$-glucosidase inhibitory activity. Compounds $3-5$ and 11 showed significant activity against $\alpha$-glucosidase with $\mathrm{IC}_{50}$ values ranging from 9.9-56.8 $\mu \mathrm{M}$. Structure-activity-relationship studies revealed that the carboxylic group plays a crucial role among all. Kinetics studies on the active compounds $\mathbf{3} \mathbf{- 5}$ and $\mathbf{1 1}$ were carried out to investigate their mechanism (mode of inhibition and dissociation constants $K_{i}$ ). All compounds were found to be non-competitive inhibitors with $K_{i}$ values in the range of 7.05 $\pm 0.17-51.15 \pm 0.25 \mu \mathrm{M}$. Moreover, in silico docking study was performed to see the allosteric hotspot for ligand binding which is targeted by our active compounds investigates the binding mode of active compounds and it was identified that compounds preferentially bind in the allosteric binding sites of $\alpha$-glucosidase. The results obtained from docking study suggested that carboxylic group is responsible for their biologic activities. To the best knowledge, this is the first report on the phytochemical investigation of $B$. elongata. In addition, the $\alpha$-glucosidase inhibition potential of all the active compounds is reported here for the first time.

Supplementary Materials: The following are available online at http://www.mdpi.com/2218-273X/10/5/751/s1, NMR and MS data for compounds 1-21 is included. This material is available free of charge via MDPI website. Table S1: Docking scores and binding interactions of compounds 3-5 and $\mathbf{1 1}$ on the predicted allosteric sites.

Author Contributions: M.A.-A. and K.R. isolated all these compounds. N.U.R. performed structural elucidation and wrote original draft of the manuscript. M.K. and A.K. conducted $\alpha$-glucosidase inhibition and kinetic study of the compounds. S.A.H. performed molecular docking studies. A.A.-R., R.C., and A.A.-H. assisted in reviewing and editing the manuscript. All authors have read and agreed to the published version of the manuscript.

Funding: The project was supported by grant from The Oman Research Council (TRC) through the funded project (BFP/RGP/CBS/18/011).

Acknowledgments: The authors are thankful to the University of Nizwa, Oman for the generous support of this research work. The authors are also thankful to Mohammed Khalifa for providing B. elongata resin and Labib Noman for the identification.

Conflicts of Interest: All authors declare that they no conflict of interest concerning this publication. 


\section{References}

1. Hudson, B.I.; Hofmann, M.A.; Bucciarelli, L.; Wendt, T.; Moser, B.; Lu, Y.; Qu, W.; Stern, D.M.; D'Agati, V.; Du Yan, S. Glycation and diabetes: The RAGE connection. Curr. Sci. 2002, 83, 1515-1521.

2. Alberti, K.G.M.M.; Zimmet, P.Z. Definition, diagnosis and classification of diabetes mellitus and its complications. Part 1: Diagnosis and classification of diabetes mellitus. Provisional report of a WHO consultation. Diabet. Med. 1998, 15, 539-553. [CrossRef]

3. Bonora, E.; Muggeo, M. Postprandial blood glucose as a risk factor for cardiovascular disease in type II diabetes: The epidemiological evidence. Diabetologia 2001, 44, 2107-2114. [CrossRef] [PubMed]

4. Care, D.F.W. Consensus statements. Diabet. Care 2001, 24, 775-778.

5. Garg, R.; Gopal, J.; Jones, G.R. Rosiglitazone: Safety and efficacy in combination with insulin in poorly controlled type 2 diabetes mellitus patients treated with insulin alone. J. Diabetes Complicat. 2007, 21, 1-6. [CrossRef]

6. Qi, L.W.; Liu, E.H.; Chu, C.; Peng, Y.B.; Cai, H.X.; Li, P. Anti-diabetic agents from natural products-An update from 2004 to 2009. Curr. Top. Med. Chem. 2010, 10, 434-457. [CrossRef]

7. Rizvi, T.; Hussain, I.; Ali, L.; Mabood, F.; Khan, A.; Shujah, S.; Rehman, N.; Al-Harrasi, A.; Hussain, J.; Khan, A. New gorgonane sesquiterpenoid from Teucrium mascatense Boiss, as $\alpha$-glucosidase inhibitor. S. Afr. J. Bot. 2019, 124, 218-222. [CrossRef]

8. Spasov, A.A.; Babkov, D.A.; Prokhorova, T.Y.; Sturova, E.A.; Muleeva, D.R.; Demidov, M.R.; Osipov, D.V.; Osyanin, V.A.; Klimochkin, Y.N. Synthesis and biological evaluation of 2-acylbenzofuranes as novel $\alpha$-glucosidase inhibitors with hypoglycemic activity. Chem. Biol. Drug Des. 2017, 90, 11841189. [CrossRef]

9. Martin, A.E.; Montgomery, P.A. Acarbose: An $\alpha$-glucosidase inhibitor. Am. J. Health Syst. Pharm. 1996, 53, 2277-2290. [CrossRef]

10. Chougale, A.D.; Ghadyale, V.A.; Panaskar, S.N.; Arvindekar, A.U. Alpha glucosidase inhibition by stem extract of Tinospora cordifolia. J. Enzyme Inhib. Med. Chem. 2009, 24, 998-1001. [CrossRef]

11. Rehman, N.U.; Khan, A.; Al-Harrasi, A.; Hussain, H.; Wadood, A.; Riaz, M.; Al-Abri, Z. New $\alpha$-glucosidase inhibitors from the resins of Boswellia species with structure-glucosidase activity and molecular docking studies. Bioorg. Chem. 2018, 79, 27-33. [CrossRef] [PubMed]

12. Ali, A.; Nasser, A.; Wurster, M.; Arnold, N.; Teichert, A.; Schmidt, J.; Lindequist, U.; Wessjohann, L. Chemical Composition and Biological Activities of Essential Oils from the Oleogum Resins of Three Endemic Soqotraen Boswellia Species. Rec. Nat. Prod. 2008, 2.

13. Alwashli, A.; Al-sobarry, M.D.; Alnamer, R.; Cherrah, Y.; Alaoui, K. Analgesic and Anti-inflammatory Activities of Boswellia elongata balf Methanolic Extracts, as Endemic Plants in Yemen. J. Biol. Act. Prod. Nat. 2012, 2, 90-98.

14. Verhoff, M.; Seitz, S.; Paul, M.; Noha, S.M.; Jauch, J.; Schuster, D.; Werz, O. Tetra-and pentacyclic triterpene acids from the ancient anti-inflammatory remedy frankincense as inhibitors of microsomal prostaglandin E2 synthase-1. J. Nat. Prod. 2014, 77, 1445-1451. [CrossRef] [PubMed]

15. Csuk, R.; Barthel-Niesen, A.; Barthel, A.; Schäfer, R.; Al-Harrasi, A. 11-Keto-boswellic acid derived amides and monodesmosidic saponins induce apoptosis in breast and cervical cancers cells. Eur. J. Med. Chem. 2015, 100, 98-105. [CrossRef] [PubMed]

16. Shah, B.A.; Qazi, G.N.; Taneja, S.C. Boswellic acids: A group of medicinally important compounds. Nat. Prod. Rep. 2009, 26, 72-89. [CrossRef]

17. Gupta, I.; Gupta, V.; Parihar, A.; Gupta, S.; Lüdtke, R.; Safayhi, H.; Ammon, H. Effects of Boswellia serrata gum resin in patients with bronchial asthma: Results of a double-blind, placebo-controlled, 6-week clinical study. Eur. J. Med. Res. 1998, 3, 511-514.

18. Gupta, I.; Parihar, A.; Malhotra, P.; Gupta, S.; Lüdtke, R.; Safayhi, H.; Ammon, H.P. Effects of gum resin of Boswellia serrata in patients with chronic colitis. Planta Med. 2001, 67, 391-395. [CrossRef]

19. Gupta, I.; Parihar, A.; Malhotra, P.; Singh, G.; Lüdtke, R.; Safayhi, H.; Ammon, H. Effects of Boswellia serrata gum resin in patients with ulcerative colitis. Eur. J. Med. Res. 1997, 2, 37-43.

20. De Sanctis, M.; Adeeb, A.; Farcomeni, A.; Patriarca, C.; Saed, A.; Attorre, F. Classification and distribution patterns of plant communities on S ocotra I sland, Yemen. Appl. Veget. Sci. 2013, 16, 148-165. [CrossRef]

21. Mothana, R.A.; Lindequist, U. Antimicrobial activity of some medicinal plants of the island Soqotra. J. Ethnopharmacol. 2005, 96, 177-181. [CrossRef] [PubMed] 
22. Mothana, R.A.; Mentel, R.; Reiss, C.; Lindequist, U. Phytochemical screening and antiviral activity of some medicinal plants from the island Soqotra. Phytother. Res. Int. J. Devoted Pharmacol. Toxicol. Eval. Nat. Prod. Deriv. 2006, 20, 298-302. [CrossRef] [PubMed]

23. Choudhary, M.I.; Shah, S.A.A.; Khan, S.N.; Khan, M.T.H. Alpha-glucosidase and tyrosinase inhibitors from fungal hydroxylation of tibolone and hydroxytibolones. Steroids 2010, 75, 956-966. [CrossRef] [PubMed]

24. MOE. Molecular Operating Environment (MOE) 2014.09; MOE: Montreal, QC, Canada, 2014.

25. Avula, S.K.; Khan, A.; Halim, S.A.; Al-Abri, Z.; Anwar, M.U.; Al-Rawahi, A.; Csuk, R.; Al-Harrasi, A. Synthesis of novel (R)-4-fluorophenyl-1H-1, 2, 3-triazoles: A new class of $\alpha$-glucosidase inhibitors. Bioorg. Chem. 2019, 91, 103182. [CrossRef] [PubMed]

26. Ur Rehman, N.; Rafiq, K.; Khan, A.; Ahsan Halim, S.; Ali, L.; Al-Saady, N.; Hilal Al-Balushi, A.; Al-Busaidi, H.K.; Al-Harrasi, A. $\alpha$-Glucosidase inhibition and molecular docking studies of natural brominated metabolites from marine macro brown Alga Dictyopteris hoytii. Mar. Drugs 2019, 17, 666. [CrossRef] [PubMed]

27. Ding, H.; Hu, X.; Xu, X.; Zhang, G.; Gong, D. Inhibitory mechanism of two allosteric inhibitors, oleanolic acid and ursolic acid on $\alpha$-glucosidase. Int. J. Biol. Macromol. 2018, 107, 1844-1855. [CrossRef]

28. Liu, Y.; Ma, L.; Chen, W.H.; Park, H.; Ke, Z.; Wang, B. Binding mechanism and synergetic effects of xanthone derivatives as noncompetitive $\alpha$-glucosidase inhibitors: A theoretical and experimental study. J. Phys. Chem. B 2013, 117, 13464-13471. [CrossRef]

29. Brindis, F.; Rodríguez, R.; Bye, R.; González-Andrade, M.; Mata, R. (Z)-3-butylidenephthalide from Ligusticum porteri, an $\alpha$-glucosidase inhibitor. J. Nat. Prod. 2011, 74, 314-320. [CrossRef]

30. Liu, T.; Yip, Y.M.; Song, L.; Feng, S.; Liu, Y.; Lai, F.; Zhang, D.; Huang, D. Inhibiting enzymatic starch digestion by the phenolic compound diboside A: A mechanistic and in silico study. Food Res. Int. 2013, 54, 595-600. [CrossRef]

31. Roig-Zamboni, V.; Cobucci-Ponzano, B.; Iacono, R.; Ferrara, M.C.; Germany, S.; Bourne, Y.; Parenti, G.; Moracci, M.; Sulzenbacher, G. Structure of human lysosomal acid $\alpha$-glucosidase-a guide for the treatment of Pompe disease. Nat. Commun. 2017, 8, 1111. [CrossRef]

32. Banno, N.; Akihisa, T.; Yasukawa, K.; Tokuda, H.; Tabata, K.; Nakamura, Y.; Nishimura, R.; Kimura, Y.; Suzuki, T. Anti-inflammatory activities of the triterpene acids from the resin of Boswellia carteri. J. Ethnopharmacol. 2006, 107, 249-253. [CrossRef] [PubMed]

33. Greve, H.L.; Kaiser, M.; Brun, R.; Schmidt, T.J. Terpenoids from the oleo-gum-resin of Boswellia serrata and their antiplasmodial effects in vitro. Planta Med. 2017, 83, 1214-1226. [CrossRef] [PubMed]

34. Paul, M. Chemotaxonomic Investigations on Resins of the Frankincense Species Boswellia papyrifera, Boswellia serrata and Boswellia sacra, Respectively, Boswellia carterii: A Qualitative and Quantitative Approach by Chromatographic and Spectroscopic Methodology. Ph.D. Thesis, Saarland University, Saarbrücken, Germany, 2012. Available online: http://scidok.sulb.unisaarland.de/volltexte/2012/4999/pdf/Dissertation_Fertig_211112. pdf (accessed on 9 November 2012).

35. Bartlett, W.R.; Johnson, W.S.; Plummer, M.S.; Small, V.R., Jr. Biomimetic polyene cyclizations. Cationic cyclization of a substrate having an internal acetylenic bond. Synthesis of euphol and tirucallol. J. Org. Chem. 1990, 55, 2215-2224. [CrossRef]

36. Zhang, P.; Jin, Y.; Chen, J.; Yao, H.; Zhang, H.; Yu, A.; Li, X. Ultrasonic-assisted nebulization extraction coupled with SPE and HPLC for the determination of triterpenoids in root of Euphorbia pekinensis Rupr. Chromatographia 2013, 76, 967-974. [CrossRef]

37. Boar, R.; Couchman, L.A.; Perkins, M.J. Synthesis of some 3-oxygenated 5alpha-Tirucalla-7, 24-and-8, 24-Dienes. J. Chem. Res. 1982, 1052-1063.

38. Itoh, T.; Tamura, T.; Matsumoto, T. Tirucalla-7, 24-dienol: A new triterpene alcohol from tea seed oil. Lipids 1976, 11, 434-441. [CrossRef]

39. Pardhy, R.; Bhattacharyya, S. Tetracyclic triterpene acids from the resin of Boswellia serrata Roxb. Indian J. Chem. 1978, 16, 174-175.

40. Al-Harrasi, A.; Ali, L.; Rehman, N.U.; Hussain, H.; Hussain, J.; Al-Rawahi, A.; Langley, G.J.; Wells, N.J.; Abbas, G. Nine triterpenes from Boswellia sacra Flückiger and their chemotaxonomic importance. Biochem. Syst. Ecol. 2013, 51, 113-116. [CrossRef]

41. Ali, L.; Hussain, J.; Al-Rawahi, A.; Al-Harrasi, A. Two New and Four Known Triterpenoids from Boswellia sacra Fluckiger. Rec. Nat. Prod. 2014, 8, 407. 
42. Ota, M.; Houghton, P.J. Boswellic acids with acetylcholinesterase inhibitory properties from frankincense. Nat. Prod. Commun. 2008, 3, 1934578X0800300105. [CrossRef]

43. Dekebo, A.; Dagne, E.; Gautun, O.R.; Aasen, A.J. Triterpenes from the resin of Boswellia neglecta. B. Chem. Soc. Ethiopia. 2002, 16, 87-90. [CrossRef]

44. Li, D.; Chen, J. Isolation and characterization of unsaturated fatty acids during bacteriorhodopsin preparation from Halobacterium halobium. Lett. Appl. Microbiol. 2007, 45, 258-261. [CrossRef] [PubMed]

45. Al-Shidhani, S.; Rehman, N.U.; Mabood, F.; Al-Broumi, M.; Hussain, H.; Hussain, J.; Csuk, R.; Al-Harrasi, A. Quantification of Incensole in Three Boswellia Species by NIR Spectroscopy Coupled with PLSR and Cross-Validation by HPLC. Phytochem Anal. 2018, 29, 300-307. [CrossRef] [PubMed]

46. Trevizan, L.N.F.; do Nascimento, K.F.; Santos, J.A.; Kassuya, C.A.L.; Cardoso, C.A.L.; do Carmo Vieira, M.; Moreira, F.M.F.; Croda, J.; Formagio, A.S.N. Anti-inflammatory, antioxidant and anti-Mycobacterium tuberculosis activity of viridiflorol: The major constituent of Allophylus edulis (A. St.-Hil., A. Juss. \& Cambess.) Radlk. J. Ethnopharmacol. 2016, 192, 510-515. [PubMed]

47. Basar, S. Phytochemical Investigations on Boswellia Species. Ph.D. Thesis, Hamburg University, Hamburg, Germany, 2005. Available online: http://ediss.sub.uni-hamburg.de/volltexte/2005/2503/pdf/DissertationSimla_Basar.pdf (accessed on 18 March 2005).

48. Ayubova, M.; Guelleh, Z.O.; Guelleh, M.O.; Brévard, H.; Baldovini, N. Analytical investigations on Boswellia occulta essential oils. Phytochemistry 2019, 164, 78-85. [CrossRef]

49. Mathe, C.; Culioli, G.; Archier, P.; Vieillescazes, C. Characterization of archaeological frankincense by gas chromatography-mass spectrometry. J. Chromatogr. A. 2004, 1023, 277-285. [CrossRef]

50. Ahn, E.K.; Oh, J.S. Lupenone isolated from Adenophora triphylla var. japonica extract inhibits adipogenic differentiation through the downregulation of PPAR $\gamma$ in 3T3-L1 cells. Phytother. Res. 2013, 27, 761-766. [CrossRef]

51. Rateb, M.E.; Ebel, R. Secondary metabolites of fungi from marine habitats. Nat. Prod. Rep. 2011, 28, $290-344$. [CrossRef]

52. Porto, C.; Ferrara, M.C.; Meli, M.; Acampora, E.; Avolio, V.; Rosa, M.; Cobucci-Ponzano, B.; Colombo, G.; Moracci, M.; Andria, G.; et al. Pharmacological enhancement of $\alpha$-glucosidase by the allosteric chaperone N-acetylcysteine. Mol. Ther. 2012, 20, 2201-2211. [CrossRef] 